Article

\title{
Mass and Energy Densities of the Cosmos
}

\author{
Ogaba Philip Obande \\ Department of Chemistry, Ahmadu Bello University, Zaria, Nigeria (Retired) \\ Email: gababands@gmail.com
}

\begin{abstract}
Fundamental principles of classical (Newtonian) physics are employed to probe the cosmological lambda $\Lambda$; it yields the values Qvac $=2.61 \times 10^{-39} \mathrm{~g} \mathrm{~cm}^{-3}$ and $\Lambda=4.78 \times 10^{-62}$. The results reveal that $\Lambda$ is a fundamental physical constant defined by vacuum density-light speed @vac ${ }^{2}$ correlation. However, the constant accelerates along the groups and periods of a universal spatial periodicity equivalent to the chemical periodicity. Previous results are cited to show that chemical elements are quantum harmonic (periodic) oscillators QHOs and their waveform oscillations exclusively define the vacuum field. A cosmological periodic unit CPU is introduced, it relies on the cosmological principle to argue that a relative physical quantity evaluated for the QHO applies to constituents of corresponding cosmological spatial quanta. Compelling evidences, backed with relevant data and quantitative expressions, are then presented to argue that: there was never a big bang, it is a Linde-universe sans "chaotic"; nature posts no singularity; mass does not curve spacetime, neither does metric space curvature trace directly to gravitation nor particle creation, gravity is classical, not quantum; reality is quadri-phasic not mono-phasic with a clear distinction between the atomic waveform defined with absolute atomic mass and condensed matter defined with relative atomic mass; every chemical element exists in three particle-generations thus, dark matter is invisible form (conjugate) of the visible element, its waveform manifests dark energy, it is not implicated in metric space expansion; Planck scale does not exist, radioactivity constrains fundamental length.
\end{abstract}

Keywords: Cosmic mass and energy densities; Dark matter and dark energy; LambdaCDM value; Metric space curvature and expansion; Vacuum field density.

\section{INTRODUCTION}

The literature leaves one in no doubt that, with reference to value of the energy density of the cosmic vacuum, theoretical physics faces a challenge of crisis proportion. The challenge is multifaceted as it touches upon the very heart of prevailing notions of physical reality from which all else derives. The literature leaves enough room to suggest that researchers may not all share a common understanding of the vacuum of space. Experimental evidence supports quantum mechanic's position that vacuous space is not Newtonian emptiness but, the question of its energy composition is far from settled. Existence of the cosmic microwave background CMB is confirmed by experiment, but quantum zero-point energy ZPE and random fluctuations of virtual particles that generate energy packets are neither well defined nor well differentiated. Above all, it is unknown whether or not dark matter/energy contribute(s) to energy content of the vacuum yet it is supposedly implicated in cosmic expansion. Kragh [1] provides an excellent starting platform from which a working knowledge obtained if one proceeded to familiarize with, amongst several others, the accounts of Carroll [2], Rugh and Zinkernagel [3], Koberinski [4], and Wesson [5]. Overduin and Wesson [6] have provided what may be regarded as the ultimate source material. From the background of these and several other sources we begin this account with a highlight of the conventional quantitative procedure for $\Lambda \mathrm{CDM}$.

\subsection{Conventional Quantitative $\Lambda \mathrm{CDM}$ Procedure}


The quantitative expressions that follow are intended only to facilitate reference, except where it is adjudged relevant, definition of terms shall be skipped. The action for general relativity in the presence of "bare" cosmological constant $\Lambda_{\mathrm{o}}$, according to Carroll, is

$$
S=\frac{1}{16 \pi G} \int d^{4} x \sqrt{-g\left(R-2 \Lambda_{o}\right)}
$$

Incidentally, (1) is normally regarded as a natural starting point for a theory of quantum gravity. Carroll explains further that classically, the effective cosmological constant is the sum of a bare term $\Lambda_{\circ}$ and the potential energy $\mathrm{V}(\phi)$; it is believed that $\mathrm{V}(\phi)$ "may change with time as the universe passes through different phases". It is also believed that quantum mechanics adds a non-trivial contribution from the zero-point energies ZPE associated with vacuum harmonic fluctuations. The potential energy of a harmonic oscillator, of course, expresses in the form $V(\phi)=1 / 2 \omega^{2} x^{2}$; for a classical oscillator this energy naturally goes to zero when $x=0$, however, for a quantum mechanical oscillator the uncertainty principle predicts the existence of a non-zero energy with the lowest state $E_{o}=1 / 2 \hbar \omega$. If a cut-off frequency $k_{\max }$ is assumed for the collection of quantized ZPE oscillators with angular speed $\omega$ in momentum space, the resulting energy density is

$$
\rho_{v a c} \sim \hbar k_{\max }^{4}
$$

Most interestingly, the energy expressed in (2) is believed to make no contribution to observed ZPE in the absence of gravity. For an infinite set of oscillators we get

$$
\rho_{v a c}=\frac{E}{V}=\frac{1}{V} \sum_{k} 1 / 2 \hbar \omega_{k} \approx \frac{\hbar}{2 \pi^{2} c^{3}} \int_{o}^{\omega_{\max }} \omega^{3} d \omega=\frac{\hbar}{2 \pi^{2} c^{3}} \omega_{\max }^{4}
$$

Equation (3) yields the following quantum field theory QFT Qvac values [6]:

$$
\begin{aligned}
& \rho_{\text {vac }}^{Q C D}=(0.3 \mathrm{GeV})^{4} \hbar^{-3} \mathrm{c}^{-5}=10^{16} \mathrm{~g} \mathrm{~cm}^{-3} ; \quad \rho_{\text {vac }}^{E W}=(200 \mathrm{GeV})^{4} \hbar^{-3} \mathrm{c}^{-5}=10^{26} \mathrm{~g} \mathrm{~cm}^{-3} \\
& \rho_{\text {vac }}^{\text {Planck }}=\left(10^{19} \mathrm{GeV}\right)^{4} \hbar^{-3} \mathrm{c}^{-5}=10^{93} \mathrm{~g} \mathrm{~cm}^{-3} ;\left|\rho_{\text {vac }}^{\text {obs }}\right| \leq\left(10^{-12} \mathrm{GeV}\right)^{4} \sim 10^{-29} \mathrm{~g} \mathrm{~cm}^{-3}
\end{aligned}
$$

The 120 order-of-magnitude disparity between $\rho_{v a c}^{\text {Planck }}$ and $\rho_{v a c}^{o b s}$ constitutes the cosmological constant puzzle CCP. Much effort has been put into attempts to tailor the predicted value with a view to making it compatible with observation, however, it would seem the efforts have been unable to realize the desired end. The preceding reflects only QFT contributions to @vac; according to [3], "In themselves these do not constitute a problem since any resulting vacuum energy in QFT may be circumvented by redefining the energy scale - only differences in vacuum energy for various configurations have experimental consequences. By contrast, GR is sensitive to an absolute value of the vacuum energy. Thus, the gravitational effect of a vacuum energy resulting from zero-point

\begin{tabular}{|c|c|c|c|c|c|c|c|c|}
\hline Vacuum & & Vacuu & zero- & QCD & luon & & The & \\
\hline $\begin{array}{l}\text { Energy } \\
\text { density }\end{array}$ & $=$ & $\begin{array}{l}\text { point energy } \\
+ \text { fluctuations }\end{array}$ & + & $\begin{array}{l}\text { and quark } \\
\text { condensates }\end{array}$ & + & $\begin{array}{l}\text { Higgs } \\
\text { field }\end{array}$ & + & $\begin{array}{l}\text { sources } \\
\text { outside }\end{array}$ \\
\hline
\end{tabular}
energies, virtual particles (higher order vacuum fluctuations), QCD condensates, fields of spontaneously broken [symmetries], and possible other, at present, unknown fields, might curve spacetime beyond recognition" (emphasis ours). Thus, in addition to quantum fields, GR is believed to make the following contributions to @vac: 
With so many contributions to Qvac from quantum fields and from GR energy sources, it is unbelievable that anyone should express surprise at the size of the divergence of calculated from observed vacuum energy density. Theorists have tried a number of tricks to "fine-tune" the calculated value but it is required that, at the minimum, the bare cosmological constant $\Lambda_{\mathrm{o}}$ would have to cancel the vacuum energy to a precision of at least 55 orders of magnitude -a tall order to meet.

We do hope the above reflects a valid highlight of the current state of establishment's research into energy density of the vacuum. Here, we present a classical perspective of the subject and attempt to account for the unusual divergence between the standard notion and the classical notion not only of the vacuum but also of reality itself.

\subsection{The Composite Reality}

We have been investigating the classical mass formula $\mathrm{h} \vartheta=\mathrm{mc}^{2}$ for some years and the effort has been turning up surprises consistently. In an attempt to simulate established relative atomic mass values, we found that reality comprises four physical states or phases composed of a waveform and three particulate matter forms one of which is our visible world; it shares same spacetime with two invisible condensed matter worlds, details of the arrangement have been reported [7, 8, 9]. The three condensed matter worlds are co-existent (collocated) and contemporaneous, they are interpenetrated by and immersed in the cosmic vacuum (waveform) field, the arrangement manifests wave-particle duality and observational three particle-generations. All four phases share common chemical periodicity and are governed by same laws of physics, they differ only in atomic mass values. Without fear of contradiction, we posit that unless this composite structure of reality is fully appreciated, particle physics would remain a merry-go-round. The wave and particulate forms are distinct and, as demonstrated in [10], both forms do not cooperate in an interaction to define an effect. Notably, neither visible nor invisible condensed matter contributes to vacuum energy content, only the vacuum field and it alone is material.

\section{Evaluation of Cosmic Parameters: the Cosmological Periodic Unit}

To our best knowledge, a clear-cut non-speculative procedure does not exist for evaluating physical properties of the cosmos, we think the chemical periodic table offers a portal, although virtual, it is the fundamental periodic unit of nature. The atom is an e-m harmonic oscillator and we have been able to evaluate the specific frequency of each element $[8,9]$; hence, in addition to (rest) mass, its other physical properties easily retrieve as simple harmonic motion SHM parameters. These properties apply to periodic quantum envelopes and indeed the whole cosmos if: i) total energy of the cosmos is strictly conserved; ii) mass-energy m-e equivalent principle is scale-invariant and holds irrevocably everywhere within the vast cosmic envelope; iii) same chemical elements define the cosmos and no where within its wide expanse does it present with a chemical element outside the established periodicity; and, iv) the cosmos is physically and thermodynamically isolated and closed as depicted in the Pan STARRS 1 "Static Sky" image [11]. With these assumptions a cosmological periodic unit CPU defines as: An arrangement of virtual or real periodic energy packets consisting, in sequence, at least sixty three elements in a periodic envelope. The number 63 comes from Russell and Russell [12]'s revelation that nature's (matter and spatial) periodicities comprise sixty three elements, the remaining elements classify with what they term "isotopes". In line with universal conformal invariance $[13,14]$ an 'element' in the present context identifies with a spacetime quantum within a periodic envelope containing hierarchical quanta sizes that may scale from the atom to the universe. An ideal example of a cosmic periodic envelope containing requisite number of elements is provided in the aligned radio galaxies ELAIS N1 [15]. Here, the word "quantum" takes its literal meaning, i.e., discrete indivisible quantity, a unit-whole or Leibniz's 'monad', not Feynman's “... peculiar aspect of nature that goes against [Newtonian physics] common sense" [16]. An envelope 
identifies with a CPU if: i) its elements' mass evolution duplicates evolution of the chemical periodicity and, ii) summation of its constituent elements' mass-energy values equals or closely approximates whole number multiple(s) of corresponding summation of the chemical periodicity. Given the cosmological principle, a relative quantity retrieved from the chemical periodicity applies to all (matter and spatial) CPUs and to the cosmos as a whole; the concept is employed to evaluate cosmic vacuum energy density.

\subsection{PROCEDURE}

Setting aside their metaphysics, we evaluate the specific oscillation frequency $\vartheta$ of the element using hints provided by Russell and Russell [12], the values are presented in [9]. Equipped with $\vartheta$ and rest mass $\mathrm{m}$ values of the atomic boson and fermion fields, i.e., $\vartheta^{*}{ }_{\mathrm{w}}, \vartheta_{\mathrm{p}}^{*}, \vartheta^{\circ} \mathrm{p}, \vartheta^{\prime} \mathrm{p}, \mathrm{m}^{*}{ }_{\mathrm{w}}, \mathrm{m}_{\mathrm{p}}^{*}, \mathrm{~m}_{\mathrm{p}}^{\mathrm{o}}$ and $\mathrm{m}_{\mathrm{p}}^{\prime}$, we evaluate vacuum energy density Qvac with the usual classical formalism. Although it is established that the Planck-Einstein-de Broglie PEB energy equation expresses atomic rest mass as

$$
m=h \vartheta / c^{2}
$$

it is unknown that $\vartheta$ and $\mathrm{c}$ are physical state or phase dependent. The vacuum transverse field $\mathrm{c}_{\mathrm{o}}=$ $2.99798458 \times 10^{8}$ differs significantly from the corresponding fermion field $\mathrm{c}^{\mathrm{o}}=3.715352291 \times 10^{-14} \mathrm{rad}$ $\mathrm{s}^{-1}$, however, the two fields identify with same quantum energy coefficient $\mathrm{h}=6.62607 \times 10^{-34} \mathrm{Js}[8,9]$, using these values density of the isolated non-bonded atom obtains from the waveform expression $[17,18]$ :

$$
\rho=6 m \vartheta^{3} / \pi c^{3}
$$

Substitution of $r=c / 2 \vartheta$ in the more familiar expression $\varrho=0.75 m / \pi r^{3}$ gives (5). The results are presented in Table I for the atom's waveform and in Tables II and III for invisible (U*p/ $\left.\mathrm{U}_{\mathrm{p}}^{\prime}\right)$ and visible $\left(\mathrm{Uo}_{\mathrm{p}}\right)$ particulate forms respectively. Definition with same chemical periodicity implies equal universe-status of the four physical phases hence the label: $\mathrm{U}^{*}{ }_{\mathrm{w}}, \mathrm{U}_{\mathrm{p}}^{*}, \mathrm{U}_{\mathrm{p}}$ and $\mathrm{U}_{\mathrm{p}}$ corresponding respectively to "Absolute ref. frame or universe", its condensed matter component, our visible world and its invisible conjugate; they work in harmony to define a common experience of single reality [8]. Classical mechanics CM identifies with quantum mechanic QM's notion of a vacuum field comprising quantum harmonic oscillators; however, $\mathrm{CM}$ differs in identifying the oscillators with waveforms of the chemical elements. Thus, e-m spectrum of the elements exclusively define vacuum content, it implies discrete m-e evolution of the CPU $[19,20]$ not continuous as is normally assumed, see, e.g., eq. (3) and [21, 22]. The cosmic value of a given relative quantity is thus summation not integration over the range of values of the 121elements of nature's chemical periodicity, for the vacuum field we have,

\subsection{RESULTS}

$$
\rho_{\mathrm{vac}}=\left(6 / \pi \mathrm{c}_{\mathrm{o}}^{2}\right) \sum_{1}^{121} \mathrm{~m}_{\mathrm{w}} \vartheta_{\mathrm{w}}^{3}
$$

The results are presented in six tables and three figures. Tables I, II, and III present densities of the atomic waveform $U^{*}$, invisible condensed matter $U_{p}^{*} / U_{p}^{\prime}$ and our visible world $U^{o}$ respectively. Table IV gives a profile of cosmic mass-energy distribution, Table V is a comparison of Planck's and nature's fundamental length and Table VI is a compilation of ratios of frequencies for a selection of elements representing the chemical periodicity. Fig. 1 is an illustration of electric and magnetic fields of single and coupled electric charges, Fig. 2 is a reproduction of NASA's NICER Experiment x-ray map of the sky and Fig. 3 is a plot of space expansion rate versus cosmological distance. Although 
the focus is primarily on vacuum energy density, it is considered relevant to investigate also density of the particulate atom.

\subsection{Lambda CMD Value}

The results give $\sum \mathrm{Q}^{*}{ }_{\mathrm{w}}=2.61 \times 10^{-39} \mathrm{~g} \mathrm{~cm}^{-3} ; \sum \mathrm{Q}_{\mathrm{p}}^{*}=9.55 \times 10^{76} \mathrm{~g} \mathrm{~cm}^{-3}$; (the two invisible condensed matter universes have equal matter density value); $\sum \mathrm{Q}^{\circ} \mathrm{p}=2.01 \times 10^{61} \mathrm{~g} \mathrm{~cm}^{-3}$. It reveals that the invisible form of the (same) atom is, on average, fifteen orders of magnitude denser than the visible form. The value $\mathrm{Qvac}=2.61 \times 10^{-39} \mathrm{~g} \mathrm{~cm}^{-3}$ gives the metric space "curvature coefficient" or energy density.

$$
\Lambda=8 \pi G \rho_{v a c} c^{-2}=4.871 \times 10^{-62} \mathrm{~cm}^{-2}
$$

\subsection{Cosmic Mass-Energy Profile}

We also investigated the cosmic mass-energy profile based on the CPU summations: $\sum \mathrm{m}^{*}{ }_{\mathrm{w}}, \sum \mathrm{m}_{\mathrm{p}}$, $\sum \mathrm{h} \vartheta^{*}{ }_{\mathrm{w}}$ and $\sum \mathrm{m}^{\mathrm{x}}{ }_{\mathrm{p}} \mathrm{C}^{\mathrm{o}}$; notably, the identity $\mathrm{h} \vartheta=\mathrm{mc}^{2}$ applies only in the vacuum field [8,9]; the results are summarized in Table IV.

Table I: Density of the atomic waveform - Absolute Ref. Frame, $\mathrm{Q}^{*}{ }_{\mathrm{w}}\left(\mathrm{kg} / \mathrm{m}^{3}\right)$

\begin{tabular}{|c|c|c|c|c|c|c|c|c|c|c|c|c|}
\hline$Z_{R}$ & atom & Density & $\mathrm{ZR}_{\mathrm{R}}$ & atom & density & $\mathrm{ZR}_{\mathrm{R}}$ & atom & density & $\mathrm{Z}_{\mathrm{R}}$ & Atom & Density & Total \\
\hline 1 & $\mathrm{Ab}(\mathrm{e})$ & $5.226 \mathrm{E}-76$ & 31 & B & $1.191 \mathrm{E}-59$ & 61 & $\mathrm{Br}$ & $1.249 \mathrm{E}-49$ & 91 & $\mathrm{~Tb}$ & $1.445 \mathrm{E}-41$ & \\
\hline 2 & $\mathrm{Bl}$ & 8.361E-75 & 32 & $\mathrm{C}$ & 3.766E-59 & 62 & $\mathrm{Kr}$ & 1.617E-49 & 92 & Dy & $1.848 \mathrm{E}-41$ & \\
\hline 3 & Bs & $1.338 \mathrm{E}-73$ & 33 & $\mathrm{~N}$ & 9.193E-59 & 63 & $\mathrm{Rb}$ & 1.617E-49 & 93 & Ho & $2.328 \mathrm{E}-41$ & \\
\hline 4 & A & $2.14 \mathrm{E}-72$ & 34 & $\mathrm{O}$ & $1.906 \mathrm{E}-58$ & 64 & Sr & $2.588 \mathrm{E}-48$ & 94 & Er & 2.897E-41 & \\
\hline 5 & $\mathrm{Jm}$ & 2.14E-72 & 35 & $\mathrm{~F}$ & $3.532 \mathrm{E}-58$ & 65 & $\mathrm{Y}$ & $1.31 \mathrm{E}-47$ & 95 & $\mathrm{Tm}$ & 3.565E-41 & \\
\hline 6 & En & 3.425E-71 & 36 & $\mathrm{Ne}$ & $6.025 \mathrm{E}-58$ & 66 & $\mathrm{Zr}$ & $4.14 \mathrm{E}-47$ & 96 & $\mathrm{Yb}$ & 4.342E-41 & \\
\hline 7 & Ey & $1.734 \mathrm{E}-70$ & 37 & $\mathrm{Na}$ & $6.025 \mathrm{E}-58$ & 67 & $\mathrm{Nb}$ & $1.011 \mathrm{E}-46$ & 97 & $\mathrm{Lu}$ & 4.342E-41 & \\
\hline 8 & $\mathrm{Ah}$ & 5.48E-70 & 38 & $\mathrm{Mg}$ & $9.64 \mathrm{E}-57$ & 68 & Mo & $2.096 \mathrm{E}-46$ & 98 & Hf & $5.415 \mathrm{E}-41$ & \\
\hline 9 & $\mathrm{Bd}$ & $1.338 \mathrm{E}-69$ & 39 & $\mathrm{Al}$ & $4.88 \mathrm{E}-56$ & 69 & Tc & $3.883 \mathrm{E}-46$ & 99 & $\mathrm{Ta}$ & 6.677E-41 & \\
\hline 10 & De & 2.774E-69 & 40 & Si & $1.542 \mathrm{E}-55$ & 70 & $\mathrm{Ru}$ & $6.625 \mathrm{E}-46$ & 100 & W & $8.148 \mathrm{E}-41$ & \\
\hline 11 & $\mathrm{Rm}$ & $5.139 \mathrm{E}-69$ & 41 & $\mathrm{P}$ & $3.766 \mathrm{E}-55$ & 71 & $\mathrm{Rh}$ & $6.625 \mathrm{E}-46$ & 101 & $\operatorname{Re}$ & $9.849 \mathrm{E}-41$ & \\
\hline 12 & $\mathrm{Bt}$ & 8.767E-69 & 42 & $S$ & 7.808E-55 & 72 & $\mathrm{Pd}$ & $1.061 \mathrm{E}-45$ & 102 & Os & $1.18 \mathrm{E}-40$ & \\
\hline 13 & Mc & 8.767E-69 & 43 & $\mathrm{Cl}$ & 1.447E-54 & 73 & $\mathrm{Ag}$ & 1.617E-45 & 103 & Ir & $1.404 \mathrm{E}-40$ & \\
\hline 14 & Pn & $1.403 \mathrm{E}-67$ & 44 & Ar & $2.468 \mathrm{E}-54$ & 74 & $\mathrm{Cd}$ & 2.368E-45 & 104 & $\mathrm{Pt}$ & $1.657 \mathrm{E}-40$ & \\
\hline 15 & $\mathrm{Vt}$ & 7.102E-67 & 45 & $\mathrm{~K}$ & $2.468 \mathrm{E}-54$ & 75 & In & 3.354E-45 & 105 & $\mathrm{Au}$ & $1.943 \mathrm{E}-40$ & \\
\hline 16 & $\mathrm{Ou}$ & $2.244 \mathrm{E}-66$ & 46 & $\mathrm{Ca}$ & 3.949E-53 & 76 & Sn & 4.619E-45 & 106 & $\mathrm{Hg}$ & $2.265 \mathrm{E}-40$ & \\
\hline 17 & $\operatorname{Tr}$ & $5.48 \mathrm{E}-66$ & 47 & Sc & 1.999E-52 & 77 & $\mathrm{Sb}$ & 4.619E-45 & 107 & $\mathrm{Tl}$ & $2.626 \mathrm{E}-40$ & \\
\hline 18 & $\mathrm{Bz}$ & $1.136 \mathrm{E}-65$ & 48 & $\mathrm{Ti}$ & 6.318E-52 & 78 & $\mathrm{Te}$ & $6.213 \mathrm{E}-45$ & 108 & $\mathrm{~Pb}$ & 3.027E-40 & \\
\hline 19 & $\mathrm{Hl}$ & 2.105E-65 & 49 & $\mathrm{~V}$ & $1.542 \mathrm{E}-51$ & 79 & I & 7.729E-45 & 109 & $\mathrm{Bi}$ & 3.027E-40 & \\
\hline 20 & G & 3.591E-65 & 50 & $\mathrm{Cr}$ & 3.198E-51 & 80 & $\mathrm{Xe}$ & $1.06 \mathrm{E}-44$ & 110 & Po & $4.072 \mathrm{E}-40$ & \\
\hline 21 & $\mathrm{Cg}$ & $3.591 \mathrm{E}-65$ & 51 & Mn & $5.925 \mathrm{E}-51$ & 81 & Cs & $1.06 \mathrm{E}-44$ & 111 & At & $5.366 \mathrm{E}-40$ & \\
\hline 22 & $\mathrm{D}$ & $5.746 \mathrm{E}-64$ & 52 & $\mathrm{Fe}$ & $1.011 \mathrm{E}-50$ & 82 & $\mathrm{Ba}$ & $1.696 \mathrm{E}-43$ & 112 & $\mathrm{Rn}$ & $6.946 \mathrm{E}-40$ & \\
\hline 23 & $\mathrm{~T}$ & 2.909E-63 & 53 & Co & 1.011E-50 & 83 & $\mathrm{La}$ & 8.585E-43 & 113 & Fr & $6.946 \mathrm{E}-40$ & \\
\hline 24 & $\mathrm{H}$ & $9.193 \mathrm{E}-63$ & 54 & $\mathrm{Ni}$ & 1.619E-50 & 84 & $\mathrm{Ce}$ & $1.391 \mathrm{E}-42$ & 114 & $\mathrm{Ra}$ & $1.111 \mathrm{E}-38$ & \\
\hline 25 & $\mathrm{~L}$ & 2.244E-62 & 55 & $\mathrm{Cu}$ & $2.468 \mathrm{E}-50$ & 85 & $\operatorname{Pr}$ & 2.139E-42 & 115 & Ac & 5.627E-38 & \\
\hline 26 & $\mathrm{Ha}$ & 4.654E-62 & 56 & $\mathrm{Zn}$ & 3.613E-50 & 86 & $\mathrm{Nd}$ & $3.156 \mathrm{E}-42$ & 116 & Th & $1.042 \mathrm{E}-37$ & \\
\hline 27 & $\mathrm{Ng}$ & $8.622 \mathrm{E}-62$ & 57 & $\mathrm{Ga}$ & 5.117E-50 & 87 & Pm & 4.497E-42 & 117 & $\mathrm{~Pa}$ & $1.778 \mathrm{E}-37$ & \\
\hline 28 & $\mathrm{He}$ & $1.471 \mathrm{E}-61$ & 58 & $\mathrm{Ge}$ & 7.048E-50 & 88 & Sm & $6.226 \mathrm{E}-42$ & 118 & $\mathrm{U}$ & $2.848 \mathrm{E}-37$ & \\
\hline
\end{tabular}




\begin{tabular}{|c|c|c|c|c|c|c|c|c|c|c|c|c|}
\hline 29 & $\mathrm{Li}$ & $1.471 \mathrm{E}-61$ & 59 & As & $7.048 \mathrm{E}-50$ & 89 & $\mathrm{Eu}$ & $8.412 \mathrm{E}-42$ & 119 & $\mathrm{~Np}$ & $4.341 \mathrm{E}-37$ & \\
\hline \multirow[t]{3}{*}{30} & $\mathrm{Be}$ & $2.353 \mathrm{E}-60$ & 60 & Se & $9.48 \mathrm{E}-50$ & 90 & $\mathrm{Gd}$ & 1.113E-41 & 120 & $\mathrm{Pu}$ & 6.356E-37 & \\
\hline & & & & & & & & & 121 & $\mathrm{Am}$ & $9.002 \mathrm{E}-37$ & \\
\hline & & $2.816 \mathrm{E}-60$ & & & 3.957E-49 & & & 3.803E-41 & & & $2.61 E-36$ & 2.61E-36 \\
\hline
\end{tabular}

Table II: Density of the element's particulate form - Rel. Component Absolute universe, $\varrho^{*} \mathrm{p} \mathrm{kg} / \mathrm{m}^{3}$

\begin{tabular}{|c|c|c|c|c|c|c|c|c|c|c|c|c|}
\hline $\mathrm{Z}_{\mathrm{R}}$ & atom & Density & $\mathrm{Z}_{\mathrm{R}}$ & atom & density & $\mathrm{Z}_{\mathrm{R}}$ & atom & density & $\mathrm{Z}_{\mathrm{R}}$ & atom & density & Total \\
\hline 1 & $\mathrm{Ab}(\mathrm{e})$ & $1.914 \mathrm{E}+34$ & 31 & B & $4.364 \mathrm{E}+50$ & 61 & $\mathrm{Br}$ & $4.575 \mathrm{E}+60$ & 91 & $\mathrm{~Tb}$ & $5.294 \mathrm{E}+68$ & \\
\hline 2 & $\mathrm{Bl}$ & $3.062 E+35$ & 32 & C & $1.379 \mathrm{E}+51$ & 62 & $\mathrm{Kr}$ & $5.923 \mathrm{E}+60$ & 92 & Dy & $6.767 \mathrm{E}+68$ & \\
\hline 3 & Bs & $4.9 \mathrm{E}+36$ & 33 & $\mathrm{~N}$ & 3.367E+51 & 63 & $\mathrm{Rb}$ & $5.923 E+60$ & 93 & Ho & $8.527 \mathrm{E}+68$ & \\
\hline 4 & A & $7.839 \mathrm{E}+37$ & 34 & $\mathrm{O}$ & $6.982 \mathrm{E}+51$ & 64 & $\mathrm{Sr}$ & $9.477 \mathrm{E}+61$ & 94 & $\mathrm{Er}$ & $1.061 \mathrm{E}+69$ & \\
\hline 5 & $\mathrm{Jm}$ & $7.839 \mathrm{E}+37$ & 35 & $\mathrm{~F}$ & $1.293 \mathrm{E}+52$ & 65 & $\mathrm{Y}$ & $4.798 \mathrm{E}+62$ & 95 & $\mathrm{Tm}$ & $1.306 \mathrm{E}+69$ & \\
\hline 6 & En & $1.254 \mathrm{E}+39$ & 36 & $\mathrm{Ne}$ & $2.207 \mathrm{E}+52$ & 66 & $\mathrm{Zr}$ & $1.516 \mathrm{E}+63$ & 96 & $\mathrm{Yb}$ & $1.59 \mathrm{E}+69$ & \\
\hline 7 & Ey & $6.35 E+39$ & 37 & $\mathrm{Na}$ & $2.207 \mathrm{E}+52$ & 67 & $\mathrm{Nb}$ & $3.702 \mathrm{E}+63$ & 97 & $\mathrm{Lu}$ & $1.59 \mathrm{E}+69$ & \\
\hline 8 & $\mathrm{Ah}$ & $2.007 \mathrm{E}+40$ & 38 & $\mathrm{Mg}$ & $3.53 E+53$ & 68 & Mo & $7.676 \mathrm{E}+63$ & 98 & Hf & $1.983 E+69$ & \\
\hline 9 & $\mathrm{Bd}$ & $4.9 \mathrm{E}+40$ & 39 & $\mathrm{Al}$ & $1.787 \mathrm{E}+54$ & 69 & Tc & $1.422 \mathrm{E}+64$ & 99 & $\mathrm{Ta}$ & $2.445 \mathrm{E}+69$ & \\
\hline 10 & De & $1.016 \mathrm{E}+41$ & 40 & Si & $5.649 \mathrm{E}+54$ & 70 & $\mathrm{Ru}$ & $2.426 \mathrm{E}+64$ & 100 & W & $2.984 \mathrm{E}+69$ & \\
\hline 11 & $\mathrm{Rm}$ & $1.882 \mathrm{E}+41$ & 41 & $\mathrm{P}$ & $1.379 \mathrm{E}+55$ & 71 & $\mathrm{Rh}$ & $2.426 \mathrm{E}+64$ & 101 & $\operatorname{Re}$ & $3.607 \mathrm{E}+69$ & \\
\hline 12 & $\mathrm{Bt}$ & $3.211 \mathrm{E}+41$ & 42 & $S$ & $2.86 \mathrm{E}+55$ & 72 & $\mathrm{Pd}$ & $3.886 \mathrm{E}+64$ & 102 & Os & $4.323 \mathrm{E}+69$ & \\
\hline 13 & Mc & $3.211 \mathrm{E}+41$ & 43 & $\mathrm{Cl}$ & $5.298 \mathrm{E}+55$ & 73 & $\mathrm{Ag}$ & $5.923 \mathrm{E}+64$ & 103 & Ir & $5.14 \mathrm{E}+69$ & \\
\hline 14 & Pn & $5.138 \mathrm{E}+42$ & 44 & Ar & $9.038 \mathrm{E}+55$ & 74 & $\mathrm{Cd}$ & $8.672 E+64$ & 104 & $\mathrm{Pt}$ & $6.069 E+69$ & \\
\hline 15 & $\mathrm{Vt}$ & $2.601 E+43$ & 45 & $\mathrm{~K}$ & $9.038 E+55$ & 75 & In & $1.228 \mathrm{E}+65$ & 105 & $\mathrm{Au}$ & $7.117 \mathrm{E}+69$ & \\
\hline 16 & $\mathrm{Ou}$ & $8.22 \mathrm{E}+43$ & 46 & $\mathrm{Ca}$ & $1.446 \mathrm{E}+57$ & 76 & Sn & $1.692 \mathrm{E}+65$ & 106 & $\mathrm{Hg}$ & $8.296 \mathrm{E}+69$ & \\
\hline 17 & $\operatorname{Tr}$ & $2.007 \mathrm{E}+44$ & 47 & $\mathrm{Sc}$ & $7.321 \mathrm{E}+57$ & 77 & $\mathrm{Sb}$ & $1.692 \mathrm{E}+65$ & 107 & $\mathrm{Tl}$ & $9.616 \mathrm{E}+69$ & \\
\hline 18 & $\mathrm{Bz}$ & $4.161 \mathrm{E}+44$ & 48 & $\mathrm{Ti}$ & $2.314 \mathrm{E}+58$ & 78 & $\mathrm{Te}$ & $2.275 \mathrm{E}+65$ & 108 & $\mathrm{~Pb}$ & $1.109 \mathrm{E}+70$ & \\
\hline 19 & $\mathrm{Hl}$ & $7.709 \mathrm{E}+44$ & 49 & $\mathrm{~V}$ & $5.649 \mathrm{E}+58$ & 79 & $\mathrm{I}$ & $2.831 \mathrm{E}+65$ & 109 & $\mathrm{Bi}$ & $1.109 \mathrm{E}+70$ & \\
\hline 20 & G & $1.315 \mathrm{E}+45$ & 50 & $\mathrm{Cr}$ & $1.171 \mathrm{E}+59$ & 80 & Xe & $3.882 E+65$ & 110 & Po & $1.491 \mathrm{E}+70$ & \\
\hline 21 & $\mathrm{Cg}$ & $1.315 \mathrm{E}+45$ & 51 & $\mathrm{Mn}$ & $2.17 E+59$ & 81 & Cs & $3.882 E+65$ & 111 & At & $1.965 \mathrm{E}+70$ & \\
\hline 22 & $\mathrm{D}$ & $2.104 \mathrm{E}+46$ & 52 & $\mathrm{Fe}$ & $3.702 E+59$ & 82 & $\mathrm{Ba}$ & $6.211 \mathrm{E}+66$ & 112 & $\mathrm{Rn}$ & $2.544 \mathrm{E}+70$ & \\
\hline 23 & $\mathrm{~T}$ & $1.065 \mathrm{E}+47$ & 53 & Co & $3.702 \mathrm{E}+59$ & 83 & $\mathrm{La}$ & $3.144 \mathrm{E}+67$ & 113 & $\mathrm{Fr}$ & $2.544 \mathrm{E}+70$ & \\
\hline 24 & $\mathrm{H}$ & $3.367 \mathrm{E}+47$ & 54 & $\mathrm{Ni}$ & $5.93 E+59$ & 84 & $\mathrm{Ce}$ & $5.094 \mathrm{E}+67$ & 114 & $\mathrm{Ra}$ & $4.07 \mathrm{E}+71$ & \\
\hline 25 & $\mathrm{~L}$ & $8.22 \mathrm{E}+47$ & 55 & $\mathrm{Cu}$ & $9.038 \mathrm{E}+59$ & 85 & $\operatorname{Pr}$ & $7.835 \mathrm{E}+67$ & 115 & Ac & $2.061 \mathrm{E}+72$ & \\
\hline 26 & $\mathrm{Ha}$ & $1.705 \mathrm{E}+48$ & 56 & $\mathrm{Zn}$ & $1.323 \mathrm{E}+60$ & 86 & $\mathrm{Nd}$ & $1.156 \mathrm{E}+68$ & 116 & Th & $3.818 \mathrm{E}+72$ & \\
\hline 27 & $\mathrm{Ng}$ & $3.158 \mathrm{E}+48$ & 57 & $\mathrm{Ga}$ & $1.874 \mathrm{E}+60$ & 87 & $\mathrm{Pm}$ & $1.647 \mathrm{E}+68$ & 117 & $\mathrm{~Pa}$ & $6.513 E+72$ & \\
\hline 28 & $\mathrm{He}$ & $5.387 \mathrm{E}+48$ & 58 & $\mathrm{Ge}$ & $2.581 \mathrm{E}+60$ & 88 & $\mathrm{Sm}$ & $2.28 \mathrm{E}+68$ & 118 & $\mathrm{U}$ & $1.043 \mathrm{E}+73$ & \\
\hline 29 & $\mathrm{Li}$ & $5.387 \mathrm{E}+48$ & 59 & As & $2.581 \mathrm{E}+60$ & 89 & $\mathrm{Eu}$ & $3.081 E+68$ & 119 & $\mathrm{~Np}$ & $1.59 \mathrm{E}+73$ & \\
\hline \multirow[t]{2}{*}{30} & $\mathrm{Be}$ & $8.619 \mathrm{E}+49$ & 60 & Se & $3.472 \mathrm{E}+60$ & 90 & $\mathrm{Gd}$ & $4.076 \mathrm{E}+68$ & 120 & $\mathrm{Pu}$ & $2.328 \mathrm{E}+73$ & \\
\hline & & & & & & & & & 121 & $\mathrm{Am}$ & $3.30 \mathrm{E}+73$ & \\
\hline \multicolumn{2}{|c|}{ Total } & $1.031 \mathrm{E}+50$ & & & $1.449 \mathrm{E}+61$ & & & $1.393 \mathrm{E}+69$ & & & $9.554 \mathrm{E}+73$ & Л.ง \\
\hline
\end{tabular}

Tble III: Density of the element's particulate form - Visible universe, $\mathrm{Q}^{\mathrm{o}} \mathrm{p} / \mathrm{kg} / \mathrm{m}^{3}$ 


\begin{tabular}{|c|c|c|c|c|c|c|c|c|c|c|c|c|}
\hline$Z_{\mathrm{R}}$ & atom & Density & $\mathrm{Z}_{\mathrm{R}}$ & Atom & density & $\mathrm{ZR}_{\mathrm{R}}$ & atom & density & $\mathrm{Z}_{\mathrm{R}}$ & atom & density & Total \\
\hline 1 & $\mathrm{Ab}(\mathrm{e})$ & $3.06 \mathrm{E}+35$ & 31 & B & $4.598 \mathrm{E}+51$ & 61 & $\mathrm{Br}$ & $1.372 \mathrm{E}+55$ & 91 & $\mathrm{~Tb}$ & $2.148 \mathrm{E}+56$ & \\
\hline 2 & $\mathrm{Bl}$ & $4.9 \mathrm{E}+36$ & 32 & C & $7.005 \mathrm{E}+51$ & 62 & $\mathrm{Kr}$ & $1.66 \mathrm{E}+55$ & 92 & Dy & $2.348 \mathrm{E}+56$ & \\
\hline 3 & Bs & $7.84 \mathrm{E}+37$ & 33 & $\mathrm{~N}$ & $1.297 \mathrm{E}+52$ & 63 & $\mathrm{Rb}$ & $1.797 \mathrm{E}+55$ & 93 & Ho & $2.491 \mathrm{E}+56$ & \\
\hline 4 & A & $1.25 \mathrm{E}+39$ & 34 & $\mathrm{O}$ & $2.201 \mathrm{E}+52$ & 64 & $\mathrm{Sr}$ & $1.984 \mathrm{E}+55$ & 94 & Er & $2.635 E+56$ & \\
\hline 5 & $\mathrm{Jm}$ & $1.25 \mathrm{E}+39$ & 35 & $\mathrm{~F}$ & $4.379 \mathrm{E}+52$ & 65 & $\mathrm{Y}$ & $2.104 \mathrm{E}+55$ & 95 & $\mathrm{Tm}$ & $2.742 \mathrm{E}+56$ & \\
\hline 6 & En & $2.01 \mathrm{E}+40$ & 36 & $\mathrm{Ne}$ & $5.584 \mathrm{E}+52$ & 66 & $\mathrm{Zr}$ & $2.331 \mathrm{E}+55$ & 96 & $\mathrm{Yb}$ & $3.019 \mathrm{E}+56$ & \\
\hline 7 & Ey & $1.02 \mathrm{E}+41$ & 37 & $\mathrm{Na}$ & $9.406 \mathrm{E}+52$ & 67 & $\mathrm{Nb}$ & $2.509 \mathrm{E}+55$ & 97 & $\mathrm{Lu}$ & $3.156 \mathrm{E}+56$ & \\
\hline 8 & $\mathrm{Ah}$ & $3.21 \mathrm{E}+41$ & 38 & $\mathrm{Mg}$ & $1.176 \mathrm{E}+53$ & 68 & Mo & $2.853 \mathrm{E}+55$ & 98 & $\mathrm{Hf}$ & $3.417 \mathrm{E}+56$ & \\
\hline 9 & $\mathrm{Bd}$ & $7.84 \mathrm{E}+41$ & 39 & $\mathrm{Al}$ & $1.784 \mathrm{E}+53$ & 69 & Tc & $3.106 \mathrm{E}+55$ & 99 & Ta & $3.61 E+56$ & \\
\hline 10 & De & $1.63 \mathrm{E}+42$ & 40 & $\mathrm{Si}$ & $2.096 \mathrm{E}+53$ & 70 & $\mathrm{Ru}$ & $3.513 \mathrm{E}+55$ & 100 & $\mathrm{~W}$ & $3.847 \mathrm{E}+56$ & \\
\hline 11 & $\mathrm{Rm}$ & $3.01 \mathrm{E}+42$ & 41 & $\mathrm{P}$ & $3.069 \mathrm{E}+53$ & 71 & $\mathrm{Rh}$ & $3.776 \mathrm{E}+55$ & 101 & $\operatorname{Re}$ & $4.048 \mathrm{E}+56$ & \\
\hline 12 & $\mathrm{Bt}$ & $5.14 \mathrm{E}+42$ & 42 & S & $3.557 \mathrm{E}+53$ & 72 & $\mathrm{Pd}$ & $4.318 \mathrm{E}+55$ & 102 & Os & $4.406 \mathrm{E}+56$ & \\
\hline 13 & Mc & $5.14 \mathrm{E}+42$ & 43 & $\mathrm{Cl}$ & $5.317 \mathrm{E}+53$ & 73 & $\mathrm{Ag}$ & $4.559 \mathrm{E}+55$ & 103 & Ir & $4.597 \mathrm{E}+56$ & \\
\hline 14 & Pn & $8.22 \mathrm{E}+43$ & 44 & $\mathrm{Ar}$ & $8.576 \mathrm{E}+53$ & 74 & $\mathrm{Cd}$ & $5.376 \mathrm{E}+55$ & 104 & $\mathrm{Pt}$ & $4.876 \mathrm{E}+56$ & \\
\hline 15 & $\mathrm{Vt}$ & $4.16 \mathrm{E}+44$ & 45 & K & $7.861 \mathrm{E}+53$ & 75 & In & $5.852 \mathrm{E}+55$ & 105 & $\mathrm{Au}$ & $5.068 \mathrm{E}+56$ & \\
\hline 16 & $\mathrm{Ou}$ & $1.32 \mathrm{E}+45$ & 46 & $\mathrm{Ca}$ & $8.688 \mathrm{E}+53$ & 76 & Sn & $6.682 \mathrm{E}+55$ & 106 & $\mathrm{Hg}$ & $5.451 \mathrm{E}+56$ & \\
\hline 17 & $\operatorname{Tr}$ & $3.21 E+45$ & 47 & Sc & $1.376 \mathrm{E}+54$ & 77 & $\mathrm{Sb}$ & $7.398 \mathrm{E}+55$ & 107 & $\mathrm{Tl}$ & $5.875 \mathrm{E}+56$ & \\
\hline 18 & $\mathrm{Bz}$ & $6.66 \mathrm{E}+45$ & 48 & $\mathrm{Ti}$ & $1.769 \mathrm{E}+54$ & 78 & $\mathrm{Te}$ & $8.926 \mathrm{E}+55$ & 108 & $\mathrm{~Pb}$ & $6.206 \mathrm{E}+56$ & \\
\hline 19 & $\mathrm{Hl}$ & $1.23 \mathrm{E}+46$ & 49 & V & $2.267 \mathrm{E}+54$ & 79 & I & $8.734 \mathrm{E}+55$ & 109 & $\mathrm{Bi}$ & $6.422 \mathrm{E}+56$ & \\
\hline 20 & G & $2.1 \mathrm{E}+46$ & 50 & $\mathrm{Cr}$ & $2.46 \mathrm{E}+54$ & 80 & $\mathrm{Xe}$ & $1 \mathrm{E}+56$ & 110 & Po & $6.424 \mathrm{E}+56$ & \\
\hline 21 & $\mathrm{Cg}$ & $2.1 \mathrm{E}+46$ & 51 & $\mathrm{Mn}$ & $3.068 \mathrm{E}+54$ & 81 & Cs & $1.051 \mathrm{E}+56$ & 111 & At & $6.548 \mathrm{E}+56$ & \\
\hline 22 & $\mathrm{D}$ & $3.37 \mathrm{E}+47$ & 52 & $\mathrm{Fe}$ & $3.276 \mathrm{E}+54$ & 82 & $\mathrm{Ba}$ & $1.198 \mathrm{E}+56$ & 112 & $\mathrm{Rn}$ & $8.178 \mathrm{E}+56$ & \\
\hline 23 & $\mathrm{~T}$ & $1.7 \mathrm{E}+48$ & 53 & Co & $4.06 \mathrm{E}+54$ & 83 & $\mathrm{La}$ & $1.254 \mathrm{E}+56$ & 113 & Fr & $8.326 \mathrm{E}+56$ & \\
\hline 24 & $\mathrm{H}$ & $5.39 \mathrm{E}+48$ & 54 & $\mathrm{Ni}$ & $3.995 \mathrm{E}+54$ & 84 & $\mathrm{Ce}$ & $1.298 \mathrm{E}+56$ & 114 & $\mathrm{Ra}$ & $8.788 \mathrm{E}+56$ & \\
\hline 25 & $\mathrm{~L}$ & $1.32 \mathrm{E}+49$ & 55 & $\mathrm{Cu}$ & $5.492 \mathrm{E}+54$ & 85 & $\operatorname{Pr}$ & $1.327 \mathrm{E}+56$ & 115 & Ac & $8.945 \mathrm{E}+56$ & \\
\hline 26 & $\mathrm{Ha}$ & $2.73 E+49$ & 56 & $\mathrm{Zn}$ & $6.152 E+54$ & 86 & $\mathrm{Nd}$ & $1.457 \mathrm{E}+56$ & 116 & Th & $9.761 \mathrm{E}+56$ & \\
\hline 27 & $\mathrm{Ng}$ & $5.05 E+49$ & 57 & Ga & $7.955 \mathrm{E}+54$ & 87 & $\mathrm{Pm}$ & $1.488 \mathrm{E}+56$ & 117 & $\mathrm{~Pa}$ & $9.594 \mathrm{E}+56$ & \\
\hline 28 & $\mathrm{He}$ & $8.62 E+49$ & 58 & Ge & $9.348 \mathrm{E}+54$ & 88 & $\mathrm{Sm}$ & $1.721 \mathrm{E}+56$ & 118 & $\mathrm{U}$ & $1.081 \mathrm{E}+57$ & \\
\hline 29 & $\mathrm{Li}$ & $7.81 \mathrm{E}+50$ & 59 & As & $1.061 \mathrm{E}+55$ & 89 & $\mathrm{Eu}$ & $1.795 \mathrm{E}+56$ & 119 & $\mathrm{~Np}$ & $1.063 \mathrm{E}+57$ & \\
\hline \multirow[t]{2}{*}{30} & Be & $2.22 \mathrm{E}+51$ & 60 & Se & $1.309 \mathrm{E}+55$ & 90 & $\mathrm{Gd}$ & $2.059 \mathrm{E}+56$ & 120 & $\mathrm{Pu}$ & $1.193 \mathrm{E}+57$ & \\
\hline & & & & & & & & & 121 & $\mathrm{Am}$ & $1.174 \mathrm{E}+57$ & \\
\hline \multicolumn{2}{|c|}{ Total } & $3.18 \mathrm{E}+51$ & & & $7.937 \mathrm{E}+55$ & & & $2.353 \mathrm{E}+57$ & & & $1.763 \mathrm{E}+58$ & $2.006 \mathrm{E}+58$ \\
\hline
\end{tabular}

4.0

\section{DISCUSSION}

The discussion focuses mainly on an attempt to account for the divergence between standard model SM's and the observational $\Lambda$ value in the light of the classical approach.

\subsection{LambdaCDM Value}

The present Qvac and $\Lambda$ values are lower than the observational upper bounds by ten orders of magnitude (OMD); it spells a far better position than the 120 OMD with use of the reigning physical model but, much more importantly, the fact that the present value results from use of an observation of nature - atomic mass - implies that it refers to the vacuum energy density as is, i.e., being based on well-established empirical data implies that the present @vac value is genetic. The result makes interesting comparison with literature. Lodge [21] reported $10^{11} \mathrm{~g} \mathrm{~cm}^{-3}$ for the then prevailing ethereal 
vacuum, he was, of course, handicapped by the same question of a cut-off frequency which plagues atomic physics to date otherwise, we see no difference between his and our approach especially as his results in a much lower divergence than the reigning approach. For his speculated primeval atom, Lemaitre [23] obtained $10^{17} \mathrm{~g} \mathrm{~cm}^{-3}$, although the value is within range of Lodge's, his approach notably marks the beginning of what culminated to the reigning paradigm. Kragh informs that from the onset Einstein made a clear distinction between $\Lambda$ value as bosonic 'property of spacetime' and its value as fermionic 'matter-energy' [1, p. 10], to date this vital distinction eludes researchers yet, it is crucial to correct visualization and realization of $\Lambda$ value, Tables I and II show clearly that atomic boson and fermion densities are worlds apart. Lodge [21] and Lemaitre [23]'s results are cited to illustrate our position that, with ref. to $\Lambda$ value, Victorian physics was not further disconnected from reality than the current physical paradigm.

\subsection{Cosmic Mass - Energy Profile}

Table IV gives: $\Sigma \mathrm{m}_{\mathrm{w}}=4.09 \times 10^{-40} \mathrm{~kg} /$ atom $/ \mathrm{CPU}$ and $\Sigma \mathrm{m}_{\mathrm{p}}=5.416 \times 10^{4} \mathrm{~kg} / \mathrm{u} / \mathrm{CPU}$ for matter's wave and particulate forms respectively. It reaffirms the reality that vacuum field's contribution to detectible cosmic mass is trivial. Invisible particulate matter commands the bulk of $99.98 \%$. For all its grandeur and patent immensity, the visible universe accounts for a negligible $0.02 \%$ of total cosmic mass (nonetheless a huge amount). On the other hand, the seemingly empty vacuum field commands a third, some $33 \%$ of total cosmic energy, while each of the two invisible particulate-matter universes share the balance $67 \%$ pro-rata leaving the visible universe again with a paltry $0.014 \%$. In other words, visible matter and energy constitute inconsequential 0.02 and $0.01 \%$ cosmic totals respectively.

Table IV: Cosmic Mass and Energy Distribution

\begin{tabular}{|c|c|c|c|c|c|}
\hline S/No. & $\begin{array}{l}\text { Ref. } \\
\text { Frame }\end{array}$ & Mass (ma/CPU) & Ratio (\%) & $\begin{array}{l}\text { Energy } \\
\text { (J/atom/CPU) }\end{array}$ & Energy Ratio (\%) \\
\hline 1 & $\mathrm{U}_{\mathrm{w}}^{*}$ & $4.088856513 \mathrm{E}-40$ & 0.0000 & 3.674880966E-23 & 32.95519 \\
\hline 2 & $\mathrm{U}_{\mathrm{p}}^{*}$ & $2.708031335 \mathrm{E}+04$ & 49.9999 & 3.738123841E-23 & 33.52234 \\
\hline 3 & $U_{p}^{\prime}$ & $2.706937435 \mathrm{E}+04$ & 49.9797 & $3.736613839 \mathrm{E}-23$ & 33.50880 \\
\hline 4 & $\Sigma \mathrm{U}_{\text {Invis. }}$ & $5.414968770 \mathrm{E}+04$ & 99.9796 & 1.114961865E-22 & 99.98633 \\
\hline 5 & $\mathrm{Uo}_{\mathrm{p}}$ & $1.104554573 \mathrm{E}+01$ & 0.0204 & $1.524709751 \mathrm{E}-26$ & 0.01367 \\
\hline 6 & Vis./Invis. & $2.039817069 \mathrm{E}-04$ & 0.0204 & 1.367499463E-04 & 0.01367 \\
\hline 7 & $\Sigma \mathrm{U}_{\text {Total }}$ & $5.416073325 \mathrm{E}+04$ & 100.00 & 1.115114336E-22 & 100.00 \\
\hline 8 & Vis./Total & $2.039401069 \mathrm{E}-04$ & 0.0204 & 1.367312483E-04 & 0.01367 \\
\hline
\end{tabular}

aUnits: $\mathrm{m}_{\mathrm{w}} /\left(\mathrm{kg}\right.$ atom $\left.{ }^{-1}\right) ; \mathrm{m}_{\mathrm{p}} / \mathrm{u}$

\subsection{Value of the Classical Cosmological Lambda}

To cross-check the present Qvac and $\Lambda$ values we slotted our results into Einstein's equation for vacuum transverse radiation, i.e.,

$$
c_{o(C M)}=\left(\left(8 \times 3.14 \times 6.67 \times 10^{-8} \times 2.61 \times 10^{-39}\right) /\left(4.87 \times 10^{-62}\right)\right)^{0.5}=3.026 \times 10^{8} \mathrm{~cm} \mathrm{~s}^{-1}
$$

The order of magnitude could not be immediately resolved, however, the reproduction motivated a re-evaluation of $\Lambda$ from the product $\mathrm{Oc}^{2}$, it turned up a pleasant surprise,

$$
\Lambda_{(C M)}=\rho_{\mathrm{vac}} \mathrm{c}_{\mathrm{o}}^{2}=2.61 \times 10^{-39} \mathrm{gcm}^{-3} \times 8.98 \times 10^{20} \mathrm{~cm}^{2} \mathrm{~s}^{-2}=2.35 \times 10^{-18} \mathrm{~g} \mathrm{~cm}^{-1} \mathrm{~s}^{-2},
$$

Notice that $\mathrm{g} \mathrm{cm}^{-1} \mathrm{~s}^{-2} \equiv \operatorname{erg~} \mathrm{cm}^{-3} \equiv$ dyne $\mathrm{cm}^{-2}$ thus, the CM approach reveals, quite unambiguously, that vacuum density correlates with vacuum transverse radiation to motivate observational lambda; it 
clearly refutes an association with curvature. The approach elucidates the dimension $\Lambda \mathrm{cm}^{-2}$ which, erstwhile, was easily prone to misunderstanding regarding exact specification of curvature's physical parameter that has the unit " $\mathrm{cm}^{-2 "}$; it is revealed here clearly unrelated to curvature. We deduce from the present and previous results that $\Lambda$ does not counter gravity, it is a fundamental physical constant achieved with a combination of other constants, it effects super-luminary bolometric radiation, i.e., metric space expansion [10]. We were quite surprised to find that $2.346 \times 10^{-18} \mathrm{~g} \mathrm{~cm}^{-1} \mathrm{~s}^{-2}$ tallies with our earlier value $\rho_{w} / \sigma_{w}=3.382^{-18}\left(\mathrm{~m}^{-1} \mathrm{~s}\right)^{2}$ giving $v_{w}=9.418 \times 10^{8} \mathrm{~m} \mathrm{~s}^{-2}$ which we have consistently attributed to metric space expansion, see section 4.4.

Compared to a minimum forty-order-magnitude difference with use of the standard procedure, the present value $2.61 \times 10^{-39} \mathrm{~g} \mathrm{~cm}^{-3}$ indicates remarkable agreement with observation. But, the key point here is confirmation that the cosmic vacuum field comprises exclusively waveforms of the familiar elements of the chemical periodicity. It manifests the CMB and, yes, it measures the zeropoint temperature associated with lowest energy state of the vacuum field. Given $\Sigma \mathrm{E}_{\mathrm{w}}=3.6749 \times 10^{-}$ ${ }^{23} \mathrm{~J}$, we have $\mathrm{T}_{\mathrm{CMB}}=\Sigma \mathrm{E}_{\mathrm{w}} / \mathrm{k}=3.6749 \times 10^{-23} \mathrm{~J} / 1.381 \times 10^{-23} \mathrm{~J} \mathrm{~K}^{-1}=2.662 \mathrm{~K}$, not far from Alpher and Herman's 5K [24] and identical to Assis and Neves' 2.7K [25], it provides quantitative evidence to reaffirm ZPE's contribution to $\Lambda$ [26]. Some of the relevant topical issues are re-examined.

Quite a number of topical issues are relevant but space and time may restrict adequate coverage; a plausible list would go as follows: 1) There was never a big bang, it is a Linde-universe sans "chaotic" [27], see Fig. 2, nature posts no singularity. 2) Mass does not curve spacetime, neither does metric space curvature trace directly to gravitation nor particle creation; gravity is classical, not quantum. 3) Reality is quadri-phasic not mono-phasic with a clear distinction between the atomic waveform defined with absolute atomic mass and condensed matter defined with relative atomic mass. 4) The three particle-generations are not unique to 'elementary' particles, it is a feature of every chemical element and dark matter happens to be invisible component of this common feature, its waveform manifests dark energy and it is not implicated in metric space expansion. 5) Planck scale does not exist, radioactivity sets the lower bound of spatial extent in atomic radius. We examine some of these subjects in a little more detail.

It feels quite uncomfortable having to fault seemingly established positions of an enormous volume of apparently exquisite contributions from some of the finest physical theorists the world has yet seen; but, often, progress necessitates painful re-structuring. What follows is submitted for critical re-examination.

\subsection{Metric Space Curvature}

Space is intrinsically curved because it comprises magnetic fields, no other reason. Electricity and magnetism are, of course, same phenomenon with different effects inside and outside mass (body). The effect is 'electric' within but 'magnetic' outside the body as illustrated in Fig. 1. Reality is electromagnetic and all bodies are coupled opposite charges. It is an electric world but observationally, unidirectional linear force-field flow inside mass attributes to 'electricity' whilst the same flow outside mass is spherical, hence 'magnetic'. Unidirectional bipolar 'electric' current gives birth to mass but spherical non-polar 'magnetic' (i.e., circular vector or scalar field) gives birth to metric space thus, as $\mathrm{m} \equiv$ energy so also space $\equiv$ magnetism but unlike mass-energy, space-magnetism inter-convertibility is infeasible because the magnetic monopole does not exist, magnetic polarity owes to electric coincidence. Metric space is essentially magnetic, it accounts for ubiquity of magnetism in cosmological bodies dating back billions of year [28] as well as in new formations [29]. Inside cosmological bodies the intense E field melts the core material and intrinsic rotation reinforces and sustains the two fields through the body's lifespan [30]. It demands review of the hydromagnetic dynamo origin of magnetic fields around cosmological bodies [31, 32, 33, 34]; the formalism is unquestionable but the concept should reflect the fact that bodies are products of electrical charge 
coupling, as shown in Fig. 1. We submit that the spherical/ellipsoidal nature of B field accounts for metric space "curvature". Indeed, 'space tells mass how to move and mass tells space how to curve' [35] but it is purely in the context of electric field coupling of two opposite (point) charges producing mass and intrinsically curved spacetime.
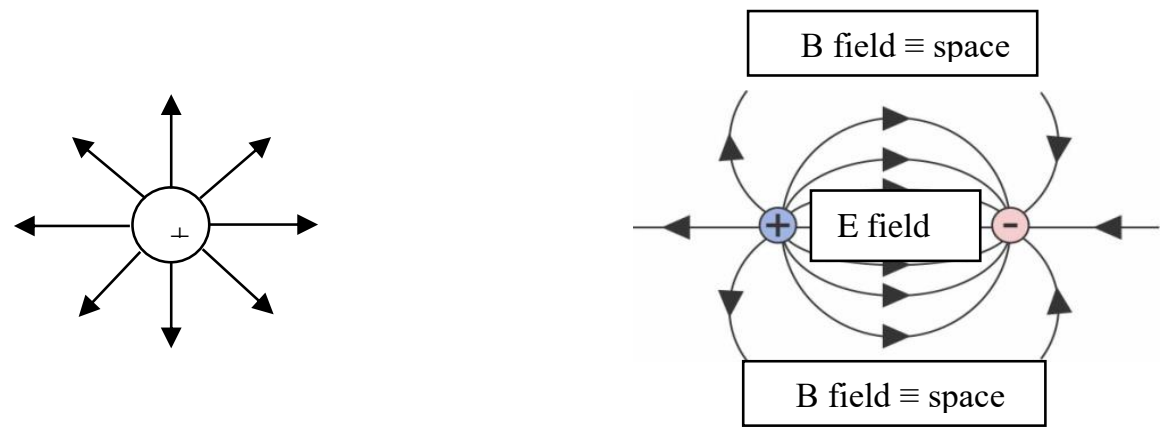

(b)

Figure 1: Electromagnetic fields: (a) electric field of an isolated positive charge; (b) internal electric and external 'magnetic' fields of coupled opposite electric point charges.

The visual image of the cosmos, the "Static Sky", [11] combines with NASA's NICER " $x$-ray map of the entire sky" [36] to create an unprecedented overall picture of our cosmic estate, reproduced below as Fig. 2 to reveal the cosmic skeleton comprising a complexly curved (electromagnetic) spacetime. According to NASA scientists, "... each bright spot represents an x-ray source while the bright filaments are their paths across the night sky". We crave indulgence of NASA's team of highly distinguished physicists and other experts to present a layman's understanding. Fig. 2 summarizes as follows: i) it depicts the internal framework or skeleton of a section of the cosmos; ii) the bright spots are (x-ray) active galactic nuclei AGN with arrangements that identify with familiar constellations and nebulae as labeled by NASA in the sister image in [36]; iii) the bright filaments or lines are condensed matter radiations of fermionic universes permeated by and enclosed within pitch black background of the cosmic vacuum field; iv) the lines (i.e., universes) number in sets of three and are mutually orthogonal, see [7];

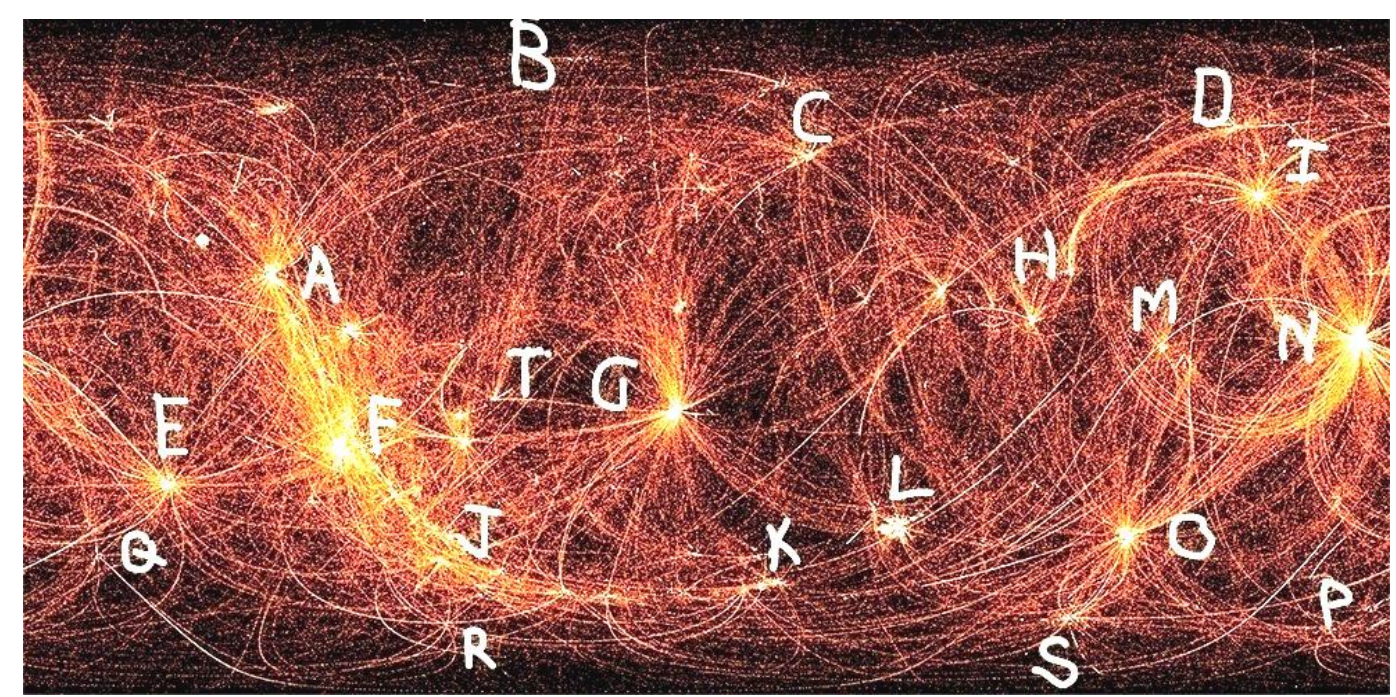

Figure 2: An X-ray map of the entire sky; posted originally by Universe.com [42]. Credit: NASANICER EXPT. (letters scripted on the original, color online). 
v) following from [7], the innermost of the set of triplet refers to our visible universe Uop with its invisible conjugate $U_{p}^{\prime}$ in the middle and invisible component $U_{p}^{*}$ of the absolute universe $U^{*}{ }_{w}$ at the outermost; vi) allowing for distortion, the triplets present with different degrees of curvature as they issue from one bright spot (AGN) to another, see, e.g., the envelopes, $A \rightarrow B, C, H, L, K J, F, A ; K \rightarrow R$; $\mathrm{D} \rightarrow \mathrm{H} / \mathrm{M}, \mathrm{N}$; triple lines to the right of $\mathrm{L}, \mathrm{C}$, curving towards $\mathrm{D} ; \mathrm{T} \rightarrow \mathrm{B} ; \mathrm{G} \rightarrow \mathrm{H}$, et cetera; vii) it is likely an internal image of a narrow strip of the "Static Sky" [11]; viii) in principle, we should have a set of coupled triplets, representing a complete periodic envelope, i.e., 6 lines streaming in and 6 lines streaming out of each bright spot, see [7]; the image, however, reveals several coupled triplets going into and out of the AGN as evident at A, E, F, G, O, N, I, we attribute the number, possibly, to presence of decaying periodic envelopes evidenced in diffuse or blurred outlines; ix) given the elemental sources of radiation [37], we posit that the background bright dots (speckles) are, amongst others, stars; $x$ ) Fig. 2 reveals much more than can be taken here, for the present purpose, it reveals unambiguously that the cosmos is essentially electrodynamical; we shall rely on Fig. 2 to attempt further account of the CCP.

\subsection{Metric Space Expansion}

An attempt to investigate metric space expansion MSE using Fig. 2 must, at present, contend with understandable obstacles: i) the conjugate wave-particle dual nature of the atom and of reality is yet unfamiliar to established physics; notably, Fig. 2's planar presentation cannot portray mutual orthogonality of the sets of three particle-generations universes to which we attribute the 'filaments'; ii) the subject is yet to advance to the state where images such as Fig. 2 might serve to elucidate key dynamics of the AGN such as that which facilitates matrix flux between universes currently tagged 'wormholes' and 'solitons' [38, 39]; iii) even after taking care of the preceding hurdles, Fig. 2 would still prove desperately inadequate for investigation of MSE for the simple reason that its coverage is rather limited; an x-ray image that reveals internal structure of an envelope spanning a hemisphere such as the Pan STARRS 1 'Static Sky' PS1-SS [11] would be invaluable. These limitations notwithstanding, we may submit as follows: i) Fig. 2 adds to confirm that the cosmos is an electrodynamical perfectly closed system, like all cosmic periodic envelopes, it is a toriod [11, 40, 41] , as a result, it can expand only locally, being restricted to within the annulus; ii) expansion reflects progression of an envelope from one symmetry group SG to another, normally from SG1 to SG IV at which point the body stops rotating, loses electric charge and becomes a potential AGN accretion candidate, see fig. 6 of [7]; iii) progression through the SGs follows a parabolic trajectory, it produces the filaments in Fig. 2 and a more careful examination would reveal that the background speckles are not exactly random, they belong to remnants of decaying or expired systems; iv) expansion presents in Fig. 2 as stepwise growth of the periodic envelope, it maps a ballooning profile as exemplified: Suppose the envelope: (1) I $\leftrightarrow \mathrm{N}$; (2) D $\rightarrow \mathrm{H} / \mathrm{M}, \mathrm{N}, \mathrm{I} \rightarrow \mathrm{D}$; (3) G $\rightarrow$ H, L $\rightarrow$ G; and (4) A $\rightarrow$ B, C, H, L, $\mathrm{K}, \mathrm{J}, \mathrm{F} \rightarrow \mathrm{A}$, define periodic envelopes, then the relative sizes $4>3>2>1$ is what manifests MSE; we think the effect would be much better defined if the image covered a hemisphere.

MSE is motivated by rotation of the body, it is a characteristic of all bodies, physical, chemical, biological or cosmological. All bodies, bosonic, fermionic, atomic, or cosmological, rotate [30], selfinteraction of the (harmonic) rotational parameters generates a number of torque fields, one of these, (8), effects expansion [10, 30] quantitatively, we have

$$
\rho_{w} / \sigma_{w}=8.51 \times 10^{-19}(\mathrm{mrad} / \mathrm{s})^{-2}
$$

Dimensional analysis gives,

$$
v_{\text {space }}=0.75 r \omega
$$

Given $\left.\mathrm{re}_{\mathrm{e}} \mathrm{w}\right)=1.499 \times 10^{8} \mathrm{~m}, \omega_{\mathrm{e}(\mathrm{w})}=6.2832^{\prime} \mathrm{m}^{\prime} \mathrm{s}^{-1}$ for the electron waveform and $\mathrm{r}(\mathrm{p})=9.1312 \times 10^{-15}$ $\mathrm{m}, \omega_{\mathrm{e}(\mathrm{p})}=12.783$ ' $\mathrm{m}^{\prime} \mathrm{s}^{-1}$ for particulate electron, (8) yields the superluminal vacuum expansion rate $\mathrm{V}_{\mathrm{w}}$ 
$=7.0639 \times 10^{8}=2.356 \mathrm{co} \mathrm{m} \mathrm{s}^{-1}$, and matter field expansion rate $\mathrm{v}_{\mathrm{p}}=8.7543 \times 10^{-14}=2.356 \mathrm{c}^{\circ} \mathrm{m} \mathrm{s}^{-1}$ where vacuum and matter transverse fields are $c_{0}=2.99792458 \times 10^{8} \mathrm{~s}^{\prime} \mathrm{s}^{-1}$ and $\mathrm{c}^{0}=3.715352291 \times 10^{-14}$ ' $\mathrm{m}^{\prime} \mathrm{s}^{-}$ ${ }^{1}$ respectively. Observe that $\mathrm{v}_{\mathrm{w}}$ is superluminal and axial not radial, i.e., a tangential $\mathrm{r}_{\mathrm{w}} \omega_{\mathrm{w}}$ velocity. We examine (9) in the context of the Hubble constant.

\subsection{The Hubble Constant}

Although (9) is a constant velocity field, a look at Fig. 2 suggests that $H_{\circ}$ necessarily varies with location and time, i.e., $H_{o}(x, t)$. Given nature's conformal invariance [13, 14], $H_{o}(x, t)$ evolution should correspond to atomic mass evolution for which, in terms of frequency, we have [20],

$$
\vartheta_{n}=\vartheta_{n-1} f(n) 8^{n}
$$

where $n=1,2,3 \ldots 10$ is the chemical period, understood here in the broader context of corresponding spacetime periodicity; there are nine periods and nine groups in nature's periodicity NP comprising six visible (known) and three invisible periods housing twenty three unknown elements preceding hydrogen; however, n's value goes up to ten in order to accommodate conventional isotopes; 8 is number of chemical groups (recall the old octet rule). It follows from (10) that Ho evolution is correspondingly segmental or quantized. As submitted earlier [11] metric expansion is a velocity field not an acceleration field, however, $\mathrm{V}_{\mathrm{w}}$ is accelerated on transiting from one conical segment (group/period) to the next as quantitatively described in (10); i.e., $\mathrm{V}_{\mathrm{w}}$ is a velocity field in spiral translation through an expanding segmental radius. The geometry is best exemplified in biology by segments of the straight shell (orthoconic Gastropoda) [42, 43]. In atomic mass evolution, the electron (waveform) executes this process starting from $\vartheta_{w(\mathrm{e})}=1.0 \mathrm{~Hz}$ to americium $\vartheta_{\mathrm{w}(\mathrm{Am})}=6.4425 \times 10^{9} \mathrm{~Hz}$; in MSE, the same process obtains with the characteristic velocity field $v_{w}=7.064 \times 10^{8} \mathrm{~m} \mathrm{~s}^{-1}$ scaled by the numerical coefficient $\vartheta_{n} / \vartheta_{n-1}$ from one group to the next; a selection of $\vartheta_{n} / \vartheta_{n-1}$ values is presented in Table $\mathrm{V}$ to reflect the periodicity. The element's intrinsic e-m frequency converts to length through the electron waveform radius $\mathrm{r}_{\mathrm{w}(\mathrm{e})}=\mathrm{c} / 2 \vartheta \times 2 \times 10^{3}=3.0 \times 10^{11} \mathrm{~m}=2 \mathrm{AU}$, a plot of $\left(\mathrm{v}_{\mathrm{w}} \vartheta_{\mathrm{n}} / \vartheta_{\mathrm{n}-1}\right) / \mathrm{m} \mathrm{s}^{-1} \mathrm{vs}$. $\mathrm{AU} / \mathrm{m}$ is presented in Fig. 3, it defines quantitatively as

$(d v / d x)_{\text {space }}={ }^{\prime \prime}{ }_{\text {space }}=5.31 A U^{0.985} \mathrm{~m} \mathrm{~s}^{-1} A U^{-1}=1.045 \times 10^{12} \mathrm{~m} \mathrm{~s}^{-1} A U^{-1}$

Eq. (11) quantifies metric space expansion but, observe that, at best, it is an approximation, Fig. 3 is not strictly linear, $\mathrm{R}^{2}=0.999$. Non-linearity results from a sudden hyperbolic jump in expansion rate to initiate spontaneous radioactivity of the quantum envelope at periodic element no. 80 equivalent in chemical periodicity to Xe, the process gradually normalizes at no.111, i.e., Ac where full-blown spontaneous decay is achieved, see [11, 17]. Notably, " $a$ "space refers to a velocity field accelerating through space not time. We should hope this fresh perspective would, if verified, inform the consistent and most commendable efforts of Reiss and his team and several others [44, 45, 46, 47], a detailed report on MSE is in progress.

Table V: A sample of $\left(\vartheta_{n} / \vartheta_{n-1}\right)$ values of elements of the chemical periodicity

\begin{tabular}{cccccccc}
\hline \hline $\mathrm{Zn}$ & Atom & Freq. $\vartheta / \mathrm{Hz}$ & $\vartheta_{\mathrm{n}} / \vartheta_{\mathrm{n}-1}$ & $\mathrm{Zn}_{\mathrm{n}}$ & Atom & Freq. $/ \mathrm{Hz}$ & $\vartheta_{\mathrm{n}} / \vartheta_{\mathrm{n}-1}$ \\
\hline 21 & $\mathrm{Cg}$ & 512 & 1 & 41 & $\mathrm{P}$ & 163840 & 1.2 \\
22 & $\mathrm{D}$ & 1024 & 2 & 42 & $\mathrm{~S}$ & 196608 & 1.1666667 \\
23 & $\mathrm{~T}$ & 1536 & 1.5 & 43 & $\mathrm{Cl}$ & 229376 & 1.1428571 \\
24 & $\mathrm{H}$ & 2048 & 1.3333333 & 44 & $\mathrm{Ar}$ & 262144 & 1 \\
25 & $\mathrm{~L}$ & 2560 & 1.25 & 45 & $\mathrm{~K}$ & 262144 & 2 \\
26 & $\mathrm{Ha}$ & 3072 & 1.2 & 46 & $\mathrm{Ca}$ & 524288 & 1.5 \\
27 & $\mathrm{Ng}$ & 3584 & 1.1666667 & 47 & $\mathrm{Sc}$ & 786432 & 1.3333333 \\
28 & $\mathrm{He}$ & 4096 & 1.1428571 & 48 & $\mathrm{Ti}$ & 1048576 & 1.25
\end{tabular}




\begin{tabular}{lccccccc}
29 & $\mathrm{Li}$ & 4096 & 1 & 49 & $\mathrm{~V}$ & 1310720 & 1.2 \\
30 & $\mathrm{Be}$ & 8192 & 2 & 50 & $\mathrm{Cr}$ & 1572864 & 1.1666667 \\
31 & $\mathrm{~B}$ & 12288 & 1.5 & 51 & $\mathrm{Mn}$ & 1835008 & 1.1428571 \\
32 & $\mathrm{Cg}$ & 16348 & 1.3304036 & 52 & $\mathrm{Fe}$ & 2097152 & 1 \\
33 & $\mathrm{Ng}$ & 20480 & 1.2527526 & 53 & $\mathrm{Co}$ & 2097152 & 1.125 \\
34 & $\mathrm{O}$ & 24576 & 1.2 & 54 & $\mathrm{Ni}$ & 2359296 & 1.1111111 \\
35 & $\mathrm{~F}$ & 28672 & 1.1666667 & 55 & $\mathrm{Cu}$ & 2621440 & 1.1 \\
36 & $\mathrm{Ne}$ & 32768 & 1.1428571 & 56 & $\mathrm{Zn}$ & 2883584 & 1.0909091 \\
37 & $\mathrm{Na}$ & 32768 & 1 & 57 & $\mathrm{Ga}$ & 3145728 & 1.0833333 \\
38 & $\mathrm{Mg}$ & 65536 & 2 & 58 & $\mathrm{Ge}$ & 3407872 & 1 \\
39 & $\mathrm{Al}$ & 98304 & 1.5 & 59 & $\mathrm{As}$ & 3407872 & 1.0769231 \\
40 & $\mathrm{Si}$ & 131072 & 1.3333333 & 60 & $\mathrm{Sc}$ & 3670016 & 1.0714286 \\
\hline
\end{tabular}

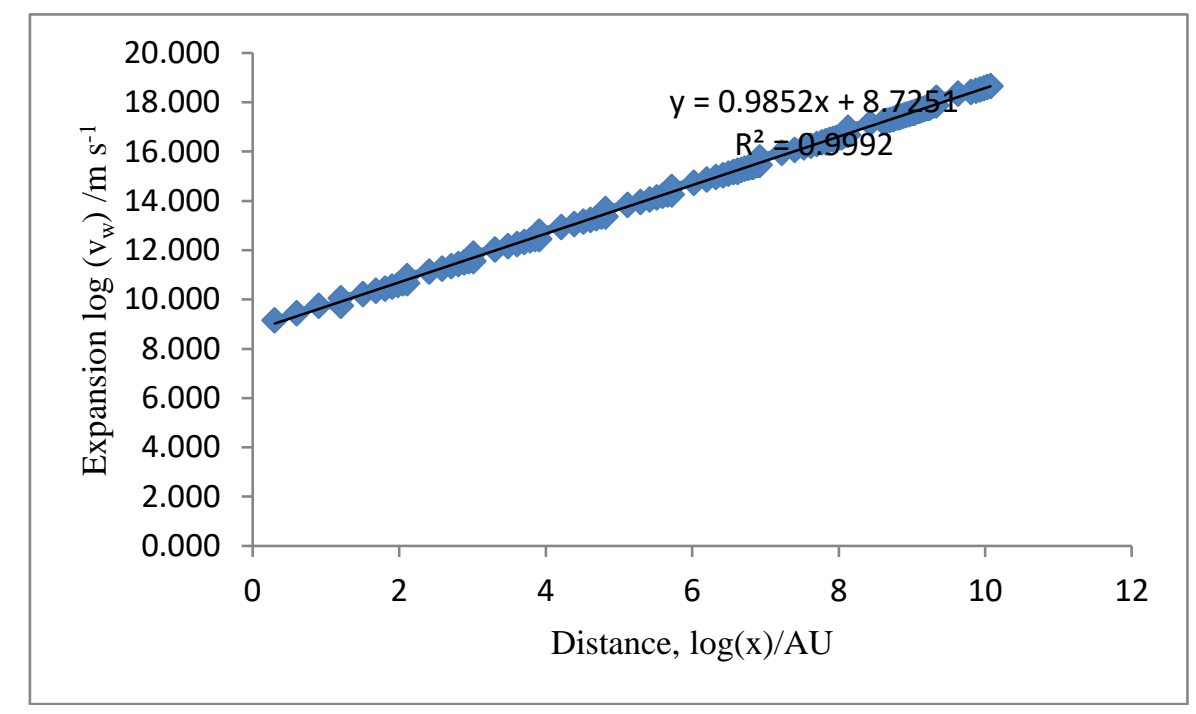

Fig. 3: Graph of space expansion rate, $\log \left(\mathrm{v}_{\mathrm{w}}\right) / \mathrm{m} \mathrm{s}^{-1} \mathrm{vs}$. distance, $\log (\mathrm{x}) / \mathrm{AU}$

\subsection{Vacuum Fluctuation, Gravitation and Matter Creation}

\subsubsection{Vacuum Fluctuation and Matter Creation}

Our literature survey was unable to locate a report on details of the energetics of vacuum fluctuation VF. The concept relies heavily on Heisenberg's uncertainty principle to argue in support of perpetual spontaneous vacuum evolution (creation) of particle-antiparticle pairs. It is attributed to non-commutation of the particle number operator and the energy operator leading to a non-zero vacuum state populated by a quantum superposition of an infinite series of eigenstates with $0,1,2$, $3, \ldots$ To set the records straight, Parker [48] points out that "... the pair creation of scalar particles from vacuum was first discovered in my PhD thesis". Evoking Heisenberg to account for VF seems far-fetched, unconvincing and unphysical. Our doubt stems from the following: 1) Uncertainty principle normally applies to the relativistic domain, it would imply that VF is relativistic and that is quite arguable. 2) Matter does indeed evolve from the vacuum but the process involves e-m field 'condensation' not scintillation. 3) Chemical kinetics associates spontaneity with exothermicity implying that VF should heat up the universe possibly to levels inimical to organic life. 4) Although several highly respected authorities have argued in favor of matter creation [1, p. 17] and some, indeed, actively promote the concept [49] the principles of foundational physics remain immutable: "matter can neither be created nor destroyed", to which we must add 'in any transformation 
whatsoever be it chemical, physical or nuclear. 5) Spontaneous evolution of one form of energy requires input of another, we are unaware of an explicit account of how the vacuum realizes the initiating energy of VF. Finally, it is difficult to see how the electric lines of force (filaments) in Fig. 2 speak in favor of cosmic VF, were those lines absent, the (stellar!) speckles at the background might somehow argue in favor of VF but, even then, they would be expected to have infrared not ultraviolet (x-ray) signature.

\subsubsection{Gravitation}

We have touched upon the mechanism (phenomenology) whereby attraction between two bodies achieves [10]. Contrary to appearances, assumption and speculation, we found that gravitation $\mathrm{G}$ is defined not by one but two potentials,

and

$$
G_{1}=r_{w} \rho_{w}^{0.25}=2.291 \times 10^{-11}
$$

$$
G_{2}=\rho_{w} \sigma_{w}^{1.333}=2.754 \times 10^{-11}
$$

Dimensional analysis gives $G_{1}=2.27 \times 10^{-11}(\mathrm{Nm})^{0.25}$ and $G_{2}=2.61 \times 10^{-11}\left(\mathrm{mr}^{2} \mathrm{\omega}\right)^{-0.333}(\mathrm{~m} /$ $s)^{-2.333}$.

Observe as follows: 1) Newtonian gravitation is a bosonic field effect, it has nothing to do with particulate matter wave. 2) The potential $\mathrm{G}_{1}$ is a pneumatic torque field, a hyperbolic outward push which increases in strength with distance and mass of the body, a 2D slice of the force field $(\mathrm{r}-\mathrm{Q}$ profile) is a perfect hyperbola [10]. Its strength degrades with distance along the polar direction $(\mathrm{r} \rightarrow$ 0 ) but reinforces with distance along the equator $(r \rightarrow \infty)$. It accounts for equatorial location of natural satellites and the observation that greater energy is required the further a satellite is removed from the nucleus also, the faster it revolves round the central body [30]. G2 is a combination of two force fields, a negative 3D angular momentum or hydrostatic pressure field $\left(m r^{2} \omega\right)^{-0.333}$ combines with a negative angular velocity $(\mathrm{m} / \mathrm{s})^{-2.333}$ to pull the gravitating bodies together, the net effect is a complex dynamic equilibrium of forces between the gravitating bodies; Mach's principle turns out cosmically valid - every body is tightly connected to all other bodies in the cosmos. Given the theoretical scenario, we see no possibility for quantum gravity, this position has been argued severally [50,51,52]. Chemical kinetics teaches that usually, spontaneous quantum charge exchange is exothermic, considering attendant geometrical re-arrangement, quantum exchange between gravitating cosmological bodies would, indeed, spell disaster because gravitation happens to be the very structural framework that secures the cosmos in equilibrium vector space.

\subsection{Fundamental Dimensions and the Dark Sector}

Fundamental dimensions are especially indispensable to energy quantification, a foundational examination of the subject therefore becomes imperative in an investigation of $\Lambda$ value.

\subsubsection{Planck Scale}

Recall one of the assumptions that underscores the CPU concept, i.e., 'there is no energy packet in the cosmos outside the elements of the chemical periodicity'. In other words, the energy scale of a conjectured spacetime packet must belong to a value locatable within values of internal energies $h \vartheta$ or $\mathrm{mc}^{2}$ of the established chemical elements. Nature's periodicity NP starts with the electron waveform at $\vartheta_{e(w)}=1 \mathrm{~Hz}$ and progresses to americium $\vartheta_{A m(w)}=6.442 \times 10^{9} \mathrm{~Hz}$ [9], the values are nature's infrared and ultraviolet cut-offs. A list of atomic radii of the chemical elements using e, $\mathrm{H}$ and Am as representative of the periodicity is presented in Table VI for comparison with corresponding Planck quantities QP1. 
Table VI: Comparison of atomic conventional physical with Planck parameters QPI

\begin{tabular}{|c|c|c|c|c|c|c|c|}
\hline \multirow{2}{*}{$\begin{array}{l}\text { Element } \\
/ Q_{P l}\end{array}$} & \multirow{2}{*}{$\begin{array}{l}\text { Fermion } \\
\mathrm{m} / \mathrm{u}\end{array}$} & \multirow{2}{*}{$\begin{array}{l}\text { Mass } \\
\text { Boson } \\
\text { m/atom }\end{array}$} & \multicolumn{2}{|c|}{ Radius (Length)/m } & \multirow{2}{*}{$\begin{array}{l}\text { Time } \\
\text { (Freq.)/s }\end{array}$} & \multicolumn{2}{|c|}{ Energy/J } \\
\hline & & & Fer & Boson & & mion & Boson \\
\hline $\mathrm{E}$ & $4.8 \times 10^{-7}$ & $7.4 \times 10^{-51}$ & 9.1 & 1.5 & 1.00 & $0^{-33}$ & $6.7 \times 10^{-34}$ \\
\hline $\mathrm{H}$ & $1.0 \times 10^{-3}$ & $1.5 \times 10^{-44}$ & $4.5 \times 10$ & $7.3 \times 10^{4}$ & $2.1 \times 10^{3}$ & $2.8 \times 10^{-30}$ & $1.4 \times 10^{-30}$ \\
\hline $\mathrm{Am}$ & $2.4 \times 10^{-1}$ & $4.7 \times 10^{-41}$ & $3.7 \times 10^{-20}$ & $2.3 \times 10^{-2}$ & $6.4 \times 10^{9}$ & $8.6 \times 10^{-24}$ & $4.3 \times 10^{-24}$ \\
\hline QPI & $2.2 \times 10^{-8}$ & - & $1.6 \times 10^{-35}$ & - & $1.9 \times 10^{43}$ & $1.9 \times 10^{9}$ & - \\
\hline
\end{tabular}

For most investigations the three particle-generations may be lumped to simplify the atom into boson (wave) and fermion (particle) fields only, as shown in Table VI, the atomic parameter is phase specific. His invaluable contribution to successful evaluation of the fundamental energy unit encouraged Max Planck to lay the foundation for derived fundamental units of mass, length and time, sadly, the results contradict nature and so, apart from his energy coefficient, i.e., the Planck Constant $h$, all other Planck parameters are misleading as evident in Table VI.

Experiment after experiment has failed to locate a lower energy packet than the electron, implying its absolute fundamentality; it is, indeed, a full-fledged element and occupies the premier position in nature's periodic order [8,9]. Thus, electron's waveform: $m_{e(w)}=7.3725 \times 10^{-51} \mathrm{~kg}$ is fundamental mass; $E_{e(w)}=6.6261 \times 10^{-34} \mathrm{~J}$ is fundamental energy and $T_{e(w)}=1 \mathrm{~s}$ is fundamental time. Notably, nature understands fundamental length only in the context of cosmological not atomic unit hence, going by electron parameters, fundamental length reads $L_{e(w)}=1.499 \times 10^{8} \mathrm{~m}=10^{-3} \mathrm{AU}$. We briefly outline some impact of Planck Scale QPI on theoretical physics and cosmology: 1) Planck dimensions: Table VI identifies QPI with fermionic matter and dimensions that measure in molar units thus, they cannot contribute to vacuum field parameters - the two fields are mutually exclusive. 2) Planck mass: literature $M_{P l}$ is only an order of magnitude lower than $m_{e}$ however, nature does not post an energy packet lower than $m_{e}$ (such a packet is yet to emerge in an experiment), we conclude therefore that literature $M_{P l}$ does not refer to fundamental mass in nature. Consistency requires $M_{P l}=$ $m_{e(w)}=7.3725 \times 10^{-51} \mathrm{~kg}$, i.e., $\left.M_{P l} \times \mathrm{c}^{2}=E_{P l}=\mathrm{hs}=6.62607 \times 10^{-34} \mathrm{~J} .3\right)$ Planckenergy: As currently defined, literature $E_{P l}$ readily emerges from literature $M_{P l}$, however, the value contradicts the celebrated results of black body radiation whence fundamental energy defines. Interestingly, physicists use $h$ everyday to probe nature but it would seem none bothers to crosscheck the identity $M_{P l} \times \mathrm{c}^{2}=\mathrm{h}$. Cosmologists seem content with use of literature $M_{P l}$ as it gives a (false) notion of an initial quantum state. 4) Planck time: Nature expresses not in time modulus but in its inverse, i.e., oscillation thus, the time scales in Table VI, including $T_{P l}$, are inverse values or frequencies - everything in nature expresses in cycles. The value $\mathrm{T}_{\mathrm{Pl}}=5.391 \times 10^{-44} \mathrm{~s}$ creates the false impression of a possibility for $\mathrm{T}_{\mathrm{o}} \rightarrow$ 0 but there is no such thing, nature exists in endless cycles. 5) Planck length: Electron waveform radius $\mathrm{r}_{\mathrm{w}(\mathrm{e})}=\lambda_{\text {photon }}=\mathrm{c} / 2 \theta=1.499 \times 10^{8} \mathrm{~m}$ is nature's fundamental length FL. It would seem FL defines conventionally as smallest "detectible" spatial extent $[53,54]$ but nature's smallest spatial extent is posted by atomic americium fermionic radius $\mathrm{r}_{\mathrm{Am}(\mathrm{p})}=3.7 \times 10^{-20} \mathrm{~m}$; notably, it is 14 orders-ofmagnitude higher than $L_{P l}$. We observe that $\mathrm{r}_{\mathrm{w}} \sim 10^{-20} \mathrm{~m}$ marks spatial dimensional lower bound below which internal stress (pressure $\sigma / \mathrm{Pa}$ ) causes the atom to 'leak' in spontaneous radioactivity [11, 17], no stable natural energy packet exists below $10^{-20} \mathrm{~m}$ radius.

In passing, we call attention to atomic americium waveform frequency $\vartheta_{A m(w)}=$ $6.442451 \times 10^{9} \mathrm{~Hz}$, it seems curious that $2 \vartheta_{\mathrm{Am}(\mathrm{w})}=12.9 \times 10^{9} \mathrm{~s}^{-1} \equiv$ 'age' $^{\prime}$ of the observable universe, we think the correspondence might hold some vital clue for physics with respect to an innate time relativity that refutes attaching an (absolute) age to the universe; notice that the equivalence informs the frequency - metric space transformation yielding Fig. 3. 


\subsubsection{Dark Matter and Dark Energy}

We have consistently identified invisible components of the three particle-generations with particulate dark matter. It is easy to verify. An attempt to simulate established relative atomic mass value invariably reveals a need for a lever system as the simplest formalism. The lever has as fulcrum the invisible 'Absolute' ref. frame $U_{w}$ above which is our visible ref. frame $U^{\circ}{ }_{p}$ and stability achieves with two invisible fermion ref. frames comprising the condensed matter component of the absolute frame $U_{p}^{*}$ and our particulate matter conjugate $U_{p}^{\prime}$ at its ends. Each ref. frame accords with a universe as all four identify with same chemical periodicity and laws of physics, they differ only in atomic mass values [8, 9]. Given the assigned radiation sources in Table I of [37], elements of the two particulate-matter invisible (dark) sector comprising $U_{\mathrm{p}}^{*}$ and $\mathrm{U}^{\prime}$ identify with charged species $[7,55]$ and high energy $\gamma, \alpha$ radiations [56,57] of the familiar chemical elements. Since same chemical elements define the visible and dark sectors we expect the invisible to have corresponding chemical, geological, biological and cosmological formations as the visible world hence, the search for dark matter should not focus on a single 'dark' energy packet but on analogues of the familiar chemical elements and structural forms in our visible frame [58]. All matter, including ourselves, comprises inseparable visible and invisible forms of the chemical element. The dark sector is invisible for only one reason - all three particle-generations worlds are mutually orthogonal, orthogonality seems to confer invisibility by hindering cross-world light passage within an otherwise common reality. All three worlds are contemporaneous and collocated, indeed, we sense our invisible companions' gravitational tug but we can't see them, apparently not with visible light. We find, therefore, a puzzle that makes "spooky action at a distance" much more familiar than baseball. Notably, in view of its particulate nature, neither DM nor DE contributes to $\Lambda$ or space expansion $[59,60]$; the label $\Lambda$ CDM is grossly misleading, it should read $\Lambda \mathrm{CMB}$ or $\Lambda \mathrm{ZPE}$.

\subsection{Plausible Estimate of Cosmic Dimensions}

From analysis of cosmological implications of his general theory of relativity GR, Einstein [1, p.22] relates the mass $M$ and volume of the universe to the radius $R$ of curvature as

$$
\mathrm{M}=2 \pi^{2} \mathrm{QR} \mathrm{R}^{3}
$$

The present result satisfies part of the requirement that $\rho_{v a c}<0, p_{\text {matter }}=0$ and $\Lambda>0$ for which

$$
R^{2}=1 / \Lambda
$$

The present $\Lambda$ value gives $R=\left(1 / 4.78 \times 10^{-62} \mathrm{~cm}^{-2}\right)^{0.5}=4.574 \times 10^{27} \mathrm{~m}$; further substitution into (14) gives $\mathrm{M}_{\mathrm{vac}}=4.9 \times 10^{48} \mathrm{~kg}$ which, of course, refers to total cosmic vacuum mass, for corresponding matter mass we use $\varrho=9.6 \times 10^{73} \mathrm{~kg} \mathrm{~m}^{-3}$ to get $1.8 \times 10^{140} \mathrm{~kg}$, this value is in error in proportion to the fraction of the vacuum occupied by matter which we assume to be a millionth (ca. 10-6). On the other hand the procedure leading to (11) adopts the cosmology convention that identifies the AU with electron waveform radius, i.e., $1.0 \mathrm{AU}=2 \times 10^{3} \mathrm{r}_{\mathrm{w}(\mathrm{e})}=2 \times 1.5 \times 10^{8} \times 10^{3}=3 \times 10^{11} \mathrm{~m}$; the figure multiplies by frequency of the last element of nature's periodicity to give radius of the $\operatorname{cosmos} R=r_{w}(A m) \times A U=$ $6.4425 \times 10^{9} \times 3 \times 10^{11} \mathrm{~m}=1.9 \times 10^{21} \mathrm{~m}$. It evaluates cosmic intangible and tangible mass as:

$$
m_{v a c}=\frac{4 \pi}{3} R^{3} \rho_{v a c}=7.5 \times 10^{28} \mathrm{~kg}
$$


Tangible matter occupies an insignificant fraction of the visible world, if we assume similar fractional occupancy of invisible frames, we may estimate that, say, a millionth part of total vacuum space is occupied by visible and invisible condensed matter thus,

$$
m_{p t c l e}=\frac{4 \pi}{3} 10^{-6} R^{3} \rho_{p t c l e}=2.76 \times 10^{120} \mathrm{~kg}
$$

With use of the correct @vac value, we notice that Einstein's cosmic dimensions $R_{v a c}=4.6 \times 10^{27} \mathrm{~m} ; \mathrm{M}_{\mathrm{vac}}$ $=4.93 \times 10^{48}$ and $\mathrm{M}_{\text {particle }}=1.81 \times 10^{140} \mathrm{~kg}$ are all much higher than corresponding $\mathrm{CM}$ values $\mathrm{R}_{\mathrm{vac}}=1.9$ $\mathrm{x} 10^{21} \mathrm{~m} ; \mathrm{M}_{\mathrm{vac}}=7.5 \times 10^{28}$ and $\mathrm{M}_{\text {particle }}=2.8 \times 10^{120} \mathrm{~kg}$; we are unable to comment on the differences.

\section{Summary and Conclusion}

- The investigation relies on a concept of cosmological periodic unit CPU to device a procedure for evaluation of relative physical quantities of the cosmos. It yields the values $\mathrm{Qvac}=2.61 \mathrm{x}$ $10^{-39} \mathrm{~g} \mathrm{~cm}^{-3} ; \Lambda=4.78 \times 10^{-62} \mathrm{~cm}^{-2}$ and gives the following cosmic mass energy ratios: visible/invisible mass $/ \%=0.02: 99.08$; visible/invisible energy $/ \%=0.014: 99.986$ revealing that the visible universe' mass and energy content is trivial compared to corresponding invisible forms.

- Re-evaluation of the transverse vacuum radiation from the expression $\mathrm{c}_{0}=(8 \pi \mathrm{GQvac} / \Lambda)^{1 / 2} \mathrm{using}$ the present values of $Q_{\text {vac }}$ and $\Lambda$ reproduced established co value. Similar re-evaluation of $\Lambda$ from the expression $\Lambda=$ Qvacc $^{2}$ gave the unit $\Lambda\left(\mathrm{g} \mathrm{cm}^{-1} \mathrm{~s}^{-2}\right) \equiv \mathrm{erg} \mathrm{cm}^{-3} \equiv$ dyne $\mathrm{cm}^{-2}$ revealing that $\mathrm{ec}^{2}$ correlation motivates $\Lambda$ and unambiguously successful refutes any association of $\Lambda$ with metric space curvature.

- It is argued that the @vac value obtained here is the actual value since the procedure relies on well-established value of an observation of nature - atomic mass.

- The chemical periodicity indentifies with the (virtual) fundamental cosmological periodic unit $\mathrm{CPU}$, its atomic energy summation gives the vacuum field zero-point energy with $\mathrm{T}=$ $2.7 \mathrm{~K}$, it manifests the cosmic microwave background $\mathrm{CMB}$ radiation, i.e., $\mathrm{ZPE} \equiv \mathrm{CMB}$ thus, the $\mathrm{CMB}$ does not, in any way, trace to a Big Bang event.

- Compelling evidences are supported with relevant quantitative expressions to argue that: there was never a big bang; mass does not curve spacetime, neither does matrix space curvature trace directly to gravitation nor particle creation; gravity is classical not quantum; magnetic field lines manifest intrinsic scale-free (atomic to cosmic) metric space curvature; dark matter and dark energy are invisible forms (conjugates) of the visible chemical elements, they are not implicated in metric space expansion; Planck scale does not exist in nature, radioactivity constrains fundamental length to radius of the heaviest element in nature's chemical periodicity, $\operatorname{Am}_{(\mathrm{p})}=2.8 \times 10^{-20} \mathrm{~m}$; no vacuum fluctuation other than atomic waveform oscillations that exclusively define the material vacuum; Hubble constant is a fundamental physical constant, it undergoes quantum jumps (acceleration) at successive higher spatial groups and periods equivalent to the chemical periodicity.

- Use of the present values Qvac $=2.61 \times 10^{-39} \mathrm{~g} \mathrm{~cm}^{-3}$ and $\Lambda=4.78 \times 10^{-62} \mathrm{~cm}^{-2}$ yielded the cosmic dimensions $\mathrm{R}=4.6 \times 10^{27} \mathrm{~m} ; \mathrm{M}_{\mathrm{vac}}=4.9 \times 10^{48} \mathrm{~kg}$ and $\mathrm{M}_{\text {particle }}=1.8 \times 10^{140} \mathrm{~kg}$ with use of Einstein's formalism and $\mathrm{R}=1.9 \times 20^{21} \mathrm{~m} ; \mathrm{M}_{\mathrm{vac}}=7.5 \times 10^{28} \mathrm{~kg}$ and $\mathrm{M}_{\text {particle }}=2.76 \times 10^{120} \mathrm{~kg}$ with use of the present classical formalism.

The results of this investigation suggest that the reigning physical paradigm is "over-theorized and over-analyzed" thus, in spite of apparent success of the Standard Model of particle physics, it fails completely when tasked with a description of reality with specific reference to cosmic mass and energy content. This shocking experience underscores an urgent concern - a growing tendency 
whereby theorists jettison the need to seek observational relevance of results obtained through pure speculation. Indeed, the tested traditional progression of research, i.e., theoretical position (hypothesis) $\rightarrow$ experimental observation $\rightarrow$ inferences $\rightarrow$ independent confirmation(s) $\rightarrow$ conclusion

$\rightarrow$ theory, is long since abandoned in favor of a misleading shortcut: theoretical position $=$ theory. If verified, the present results would mean a wake-up call for caution and revival of indispensability of the Victorian approach. And, of course, as in previous reports, the present results proclaim primacy of the simple-looking energy equation $h \vartheta=m c^{2}$, though dismissively simple, the expression compellingly identifies with the Theory of Everything.

\section{References}

1. Kragh, H. (2014). Historical aspects of post-1850 cosmology. AIP Conf. Proc. 1632, 3 DOI: $10.1063 / 1.4902842$

2. Carroll, S. M. (2001). The Cosmological Constant. Living Rev. Relativity 4, 1; DOI: 10.12942/lrr2001-1

3. Rugh, S. E., and Zinkernagel, H. (2001). Stud. Hist. Philos. Mod. Phys. 33, 663

4. Koberinski, A. (2017). Problems with the cosmological constant problem. https://philsci-archive.pitt.edu/14244/1/problems-cosmological-constant.pdf/

5. Wesson, P. S. (undated). Zero-Point Fields, Gravitation and New Physics. Retrieved from https://calphys.com/articles/Wesson.pdf; DOI:10.1.1.36.2994

6. Overduin, J. M. and Wesson, P. S. (2004). Dark Matter and Background Light. Phys. Report. $402,276-406$

7. Obande, O. P. (2018). A field Concept of the Black Hole. Appl. Phys. Res. 10(4), 66; DOI:10.5539/apr.v10n4p66

8. Obande, O. P. (2013). Notes on Russellian Cosmogony - Part I: Absolute Atomic Mass. Int. J. Engr. Sci. 2, 68; http://www.theijes.com/papers/v2-i4/part.\%20(2)/M0242068077.pdf

9. Obande, O. P. (2015). Notes on Russellian Cosmogony. II. A procedure for theoretical evaluation of relative atomic mass and internal energy. Phys. Essays 28(1), 78; DOI: 10.4006/0836-1398-28.1.78

10. Obande, O. P. (2017). On the Fundamental Physical Constants: I. Phenomenology. Appl. Phys. Res. 9(5), 42; DOI: 10.5539/apr.v9n5p42

11. Obande, O. P. (2017). The Pan-STARRS 1 Static Sky: A Classical Physics Goldmine for Cosmology, Astrophysics and Atomic Physics. Appl. Phys. Res. 9(6), 75; DOI: 10.5539/apr.v9n6p75

12. Russell, W. and Russell, L. (1981). Atomic Suicide? 2nd ed. University of Science and Philosophy, Waynesboro, VA; http://www.scribed.com/doc/85626193/Atomic-Suicide-Walter-and-Lao$\underline{\text { Russell }}$

13. Oldershaw, R. L. (2007). Discrete Scale Relativity. Astroph. Space Sci. 311(4), 431; DOI: $10.1007 / \mathrm{s} 10509-007-9557-x$ 
14. Wikiversity. (2017). Similarity of matter levels. Retrieved from https://en.wikiversity/wiki/Similarity of matter levels

15. Taylor, A. R and Jagannathan, P. (2016). Alignments of radio galaxies in deep radio imaging of ELAIS N1. Mont. Not. Roy. Astron. Soc. Letts. 459(1), L36

16. Feynman, R. P. (2013). Lectures on Physics. See, https://www.feynmanlectures.caltech.edu

17. Obande, O. P. (2015). Classical mechanics analysis of the atomic particulate forms. Int. J. Engr. Sci. 4(6), 1; http://www.theijes.com/papers/v4-i6/version-2/A046201011.pdf

18. Obande, O. p. (2015). Classical Definitions of Gravitation, Electricity and Magnetism. Appl. Phys. Rev. 7(6), 85; DOI: 10.5539/apr.v7n6p85

19. Obande, O. P. (2016). Atomic Mass: Origin, Units and Constants. Appl. Phys. Res. 8(1), 92; DOI: 10.105539/apr.v8n1p92

20. Obande, O. P. (2016). A classical perspective of the cosmological constant. Phys. Essays 29(2), 228; DOI: 10.4006/083-1398-29.2.228

21. Lodge, O. (1907). The Density of the Aether. Phil. Mag.13, 488

22. Nernst, W. (1916). Gesellschat 18. 83

23. Lemaitre, G. (1934). Evolution of the Expanding Universe. Proc. Nat. Acad. Sci. 20, 12

24. Alpher, R. A. and Herman, R. C. (1948). Evolution of the universe. Nature 74, 774

25. Assis, A. K. T., Neves, M. C. D. (1995). History of the 2.7 K Temperature Prior to Penzias and Wilson. Apeiron 2(3), 79

26. Fujii, Y. (2014). Is the zero-point energy a source of the cosmological constant? https://doi.org/abs/1403.3749

27. Linde, A. (1986). Eternally Existing Self-Reproducing Chaotic Inflationary Universe. Phys. Lett. B175(4), 395; DOI: 10.1016/0370-2693(86)90611-8

28. Mao, S. A., Cavili, C., Gaensler, B. M. et al. (2017). Detection of Microgauss coherent magnetic fields in a galaxy five billion years old. Nature Astron. DOI: 10.1038/s41550-0170218-x

29. Francesco, F. et al. (2016). Magnetism drives stellar births. Nature 538, 8 Astron. Astrophys 593,

30. Obande, O. P. (2019). On Intrinsic Rotation of Bodies. J. High Energy Phys. Grav. and Cosm. 5, 868; DOI: 10.4236/jhepgc.2019.53043

31. Kern, J. W., Vestine, E. H. (1963). Magnetic Field of the Earth and Planets. Space Sci. Rev. 2(1), 136

32. Crowling, T. G. (1981). The Present Status of Dynamo Theory. Ann. Rev. Astrophys. 19, 115

33. Weiss, N. (2002). Dynamos in planets, stars and galaxies. Astron. Geophys. 43(3), 3.09; DOI: 10.10946/j1468-4004.2002.43309.x 
34. Danilov, V. (2016). On the Nature of the Magnetic Field of the Earth and Other Planets. Prog. Phys. 12(1), 41

35. Huggins, E. (2018). Curved Spacetime (Gravity) Tells Mass (Energy) How to move. Phys. Teacher 56, 591; DOI: 10.1119/1.5080570

36. Williams, M. (2019). NASA is building up a map of the entire sky seen in x-rays, line by line with its NICER experiment. https://www.universe.com/142392/nasa-is-building-up-a-map-ofthe-entire-sky-seen-in-x-rays-line-by-line-with-its-nicer-experiment/

37. Obande, O. P. (2016). On the Photon's Identity: Implications for Relativity and Cosmology. Appl. Phys. Res. 8(5), 10; DOI: 10.5539/apr.v8n5p10

38. Keihn, R. M. (2004). Falaco Solitons - cosmic strings in a swimming pool. https://www2.pair.com/csdc/pdf/falsol.pdf

39. Good Elf. (2016). Theories of Particles Based on Vortices. https://physicsdiscussionforum.org

40. Williamson, J. G. and van der Mark, M. B. (1997). Is the electron a photon with toriodal topology? Annals Found. Louis d Broglie 22(2). 133

41. Imanishi, M., Nakanishi, K. et al. (2018). ALMA Reveals an Inhomogeneous Compact Rotating Dense Molecular Torus at NGC 1068 Nucleus. https://arxiv.org/abs/1801.0654

42. Emiliani, C. (1995). Planet Earth. Cosmology, geology, and evolution of life and environment. Cambridge. p. 456, Fig. 21.12

43. Golubev, S. N. (2014). The Emergence and Existence of Life in the World of Elements and the Physical Vacuum. Achiv. Life Sci. 8(1), 1 DOI: 10.1016/j.als.2014.11.001

44. Reiss, A. G., Casertano, S., Yuan, W. et al. (2019). Large Magellanic Cloud Cephid Standards Provide a 1\% Foundation for the Determination of the Hubble Constant and Stronger Evidence for Physics Beyond LambdaCDM. https://arxiv.org/pdf/1903.07603.pdf

45. Reiss, A. G., Macri, L. M., Hoffmann, S. L. et al. (2016). A 2.4\% determination of the local value of the Hubble constant based on observations with the NASA/ESA Hubble Space Telescope. ApJ 826(1), 56 https://arxiv.org/pdf/1604.0124.pdf

46. Reiss, A. G., Macri, L. M., Casertano, S. et al. (2011). A 3\% solution: determination of the Hubble Constant with the $150 \times 10^{6} \mathrm{~km}$ Hubble Telescope and Wide Field Camera 3" ApJ 730(2), 119 DOI: $10.1088 / 0004-637 X / 730 / 2 / 119$

47. Birrer, S. Treu, T., Rusu, C.E. et al. (2019). HOLiCOW - IX. Cosmographic analysis of the doubly imaged quasar SDSS $1206+4332$ and a new measurement of the Hubble constant. MNRAS 484(4), 4726

48. Parker, L. (2012). Particle creation and particle number in an expanding universe. https://arxiv.org/pdf/1205.5616.pdf 
49. Hawking, S. W. (1975). Particle creation by black holes. Comm. Math. Phys. 43(3), 199; DOI: 10.10097/BF02345020

50. Carlip, S. (2001). Quantum gravity: Progress Report. Rep. Prog. Phys. 64(8). 885 DOI: $10.1088 / 034-4885 / 64 / 8 / 301$

51. Mattingly, J. (2005). Is quantum gravity necessary? In: Kox, A. J., Eisenstaedt, J. (eds.) The Universe of General Relativity, pp 327 - 338. Birkhauser, Boston.

52. Wuthrich, C. (2005). To quantize or not to quantize: fact and folklore in quantum gravity. Philos. Sci. 72(5), 777; DOI: 10.1086/508946

53. Calmet, X., Graesser, M., Hsu, S. D. (2004). Minimum length from Quantum Mechanics and General Relativity. Phys. Rev. Lett. 93, 21110; https://arxiv.org/pdfhep-th/0405033.pdf

54. Garay, L. J. (2015). Discrete or Continuous?: The Quest for Fundamental Length in Modern Physics. Phys. Today 68(1), 44; DOI: 10,1063/PT.3265

55. Borghino, D. (2015). New Study Suggests Dark Matter is Charged Particle. https://newatlas.com/stealth-dark-matter

56. Weniger, C. (2012). A Tentative Gamma-Ray Line from Dark Matter Annihilation at the Fermi Large Area Telescope. arXiv: 1204.2797v2 [hep-ph]

57. Daylan, T., Finkbeinav, D. P., Hooper, D. et al. (2014). The Characterization of the Gamma-Ray Signal from the Central Milky Way: A Compelling Case for Annihilating Dark Matter. arXiv: 1402.6703 [astro-ph-HE]

58. Jacobs, D. M. Starkman, G. D. and Lynn, B. W. (2015). Macro Dark Matter. arXiv:1410.2236; DOI: $10.1093 / \mathrm{mnras} / \mathrm{stv} 774$

59. Baltay, C. (2014). The accelerating universe and dark energy. Int. J. Mod. Phys. 23(6), 143001; DOI: $10.1142 / S 0218271814300122$

60. Wang, Q., Zhen, Z. and William, G. U. (2017). How the huge energy of quantum vacuum gravitates to drive slow accelerating expansion of the universe. Phys. Rev. D95.10, p.103504 